\title{
Rational Components of Hilbert Schemes
}

\author{
Paolo Lella $(*)$ - Margherita RogGero (*)
}

ABSTRACT - The Gröbner stratum of a monomial ideal $j$ is an affine variety that parameterizes the family of all ideals having $j$ as initial ideal (with respect to a fixed term ordering). The Gröbner strata can be equipped in a natural way with a structure of homogeneous variety and are in a close connection with Hilbert schemes of subschemes in the projective space $\boldsymbol{P}^{n}$. Using properties of the Gröbner strata we prove some sufficient conditions for the rationality of components of $\mathcal{H} \mathrm{ilb}_{p(z)}^{n}$. We show for instance that all the smooth, irreducible components in $\mathcal{H i l b}_{p(z)}^{n}$ (or in its support) and the Reeves and Stillman component $H_{R S}$ are rational. We also obtain sufficient conditions for isomorphisms between strata corresponding to pairs of ideals defining a same subscheme, that can strongly improve an explicit computation of their equations.

\section{Introduction.}

The aim of the present paper is to investigate effective methods to study the Hilbert scheme of subschemes in the projective space $\boldsymbol{P}^{n}$, both on the theoretical and the computational point of view, using Gröbner basis tools. Several authors have been working in this direction during last years (for instance [3, 21]), but our motivations mainly refer to some ideas and hints contained in the paper [19] by Notari and Spreafico. In order to get a stratification of $\mathcal{H}$ ilb $b_{p(z)}^{n}$, they introduce some affine varieties $\mathcal{S} t(\mathfrak{j})$ (here called Gröbner strata) parameterizing families of ideals in $k\left[X_{0}, \ldots, X_{n}\right]$ having the same initial ideal $j$ with respect to a fixed term ordering on the monomials. When only homogeneous ideals in $k\left[X_{0}, \ldots, X_{n}\right]$ are concerned, we write $\mathcal{S} t_{h}(\mathfrak{j})$. The ideal defining a Gröbner stratum springs out from a procedure based on Buchberger's algorithm, but involves a reduction with respect to a set of polynomials which is not a Gröbner basis.

(*) Indirizzo degli A.: Dipartimento di Matematica dell'Università di Torino, Via Carlo Alberto 10, 10123 Torino, Italy.

E-mail: paolo.lella@unito.it margherita.roggero@unito.it

Supported by PRIN (Geometria della varietà algebriche e dei loro spazi di moduli) co-financed by MIUR (2008). 
It is not difficult to realize that the support of $\mathcal{S} t(\mathrm{j})$ only depends on the initial data (the term ordering, the ideal $j$, etc.), but one cannot be beforehand sure that different choices in the reduction steps always lead to the same ideal. In other words it is not clear if Gröbner strata are schemetheoretically well defined. This is a crucial point that is underlined for instance in [21, Section 3]. In fact, if anyone wants to deduce properties of an Hilbert scheme using a Gröbner stratum, it is necessary to consider carefully the non-reduced structures, because $\mathcal{H}$ ilb $b_{p(z)}^{n}$ can have non-reduced components (see $[12,14,18]$ ). A first achievement in this paper is the following result (see Theorem 3.6):

THEOREM A. The ideal defining $\mathcal{S} t(\mathfrak{j})$ does not depend on the reduction choices.

In fact we exhibit an equivalent, but intrinsic definition for the ideal of $\mathcal{S} t(\mathfrak{j})$, which by the way also allows a great simplification in the procedure for an explicit computation of this ideal.

A meaningful property that all Gröbner strata enjoy is that they are homogeneous with respect to some non-standard grading. Homogeneous varieties are of a very special type: for instance they can be isomorphically embedded in the Zariski tangent space at the origin, which is of course the smallest affine space in which such an embedding can be done. Therefore a smooth homogeneous variety has to be isomorphic to an affine space; in fact, the variety has the same dimension as the space in which it can be embedded. Moreover one can obtain directly the ideal of $\mathcal{S} t(\mathrm{j})$ in the "minimal embedding" in the Zariski tangent space, through a preliminary detection of a maximal set of "eliminable variables" (we briefly resume this method in $\S 4$ ). This is a second key point in our work, because one of the main difficulties usually met studying Gröbner strata (and even more Hilbert schemes) is due to the huge number of variables that their equations involve, even in very simple cases. Besides the obvious computational gain, we would like to underline the interesting theoretical outcome of this method: most of our proofs are obtained just using the minimal embedding.

A second useful tool that can simplify the computation of equations defining a stratum is given in Theorem 4.7. Though the strata corresponding to ideals that define a same subscheme are in general non-isomorphic, however we show that two of them give rise to isomorphic strata when they satisfy suitable sufficient condition, so that we can equivalently take into consideration the most convenient one. 
Theorem B. Let $j$ be monomial ideal in $k\left[X_{0}, \ldots, X_{n}\right]$ which is saturated and Borel-fixed w.r.t. the order on the set of variables $X_{0} \succ X_{1} \succ \ldots \succ X_{n}$.

i) For every $m$, there is a set of eliminable variables of the ideal defining $\mathcal{S} t_{h}\left(\dot{\mathfrak{j}}_{\geqslant m}\right)$, that contains all variables except at most the ones appearing in polynomials $F$ such that $\mathrm{LT}(F)=X^{\alpha} X_{n}^{m-|\alpha|}$, where $X^{\alpha}$ is a minimal generator of $\mathrm{j}$.

ii) If $X_{n-1}$ does not appear in any monomial of degree $m+1$ in the monomial basis of $\dot{j}$, then $\mathcal{S} t_{h}\left(\dot{\mathfrak{j}}_{\geqslant m-1}\right) \simeq \mathcal{S} t_{h}\left(\dot{\mathfrak{j}}_{\geqslant m}\right)$.

iii) Especially, if $s$ is the maximal degree of monomials in the monomial basis of $\dot{j}$ containing $X_{n-1}$, then $\mathcal{S} t_{h}\left(\dot{\mathfrak{j}}_{\geqslant s-1}\right) \simeq \mathcal{S} t_{h}\left(\dot{j}_{\geqslant m}\right)$ for every $m \geq s$.

In $\S 5$ and $\S 6$ we investigate more closely the natural connection between the Gröbner stratum $\mathcal{S} t_{h}(\mathfrak{j})$ and the Hilbert scheme $\mathcal{H}$ ilb $b_{p(z)}^{n}$, where $p(z)$ is the Hilbert polynomial of $k\left[X_{0}, \ldots, X_{n}\right] / \dot{j}$. As for every ideal $\mathfrak{i}$ whose initial ideal is $\dot{\mathfrak{j}}$, the modules $k\left[X_{0}, \ldots, X_{n}\right] / \mathfrak{i}$ and $k\left[X_{0}, \ldots, X_{n}\right] / \dot{j}$ share the same Hilbert function, there is an obvious set-theoretic inclusion $\mathcal{S t}_{h}(\mathfrak{j}) \subseteq \mathcal{H}$ ilb $_{p(z)}^{n}$. However it is not a simple task to understand if this inclusion is an algebraic embedding or not. The paper [19] deals with the same question, but mainly concerns Gröbner strata of saturated ideals with respect to the term ordering DegRevLex: note that every subscheme $Z$ in $\boldsymbol{P}^{n}$ can be defined by the saturated ideal $I(Z)$. In this paper we prefer to consider a slightly different approach, modeled on the classical construction of the Hilbert schemes (see for instance [1, 11]). For every $Z \in \mathcal{H}$ ilb $b_{p(z)}^{n}$ we consider the ideal $I(Z)_{\geqslant r}$, where $r$ is the Gotzmann number of $p(z)$. As $r$ is the worst Castelnuovo-Mumford regularity for all $Z \in \mathcal{H}$ ilb $_{p(z)}^{n}$, the Gröbner strata (with respect to any term ordering) of monomial ideals generated in degree $r$ cover $\mathcal{H}$ illo $_{p(z)}^{n}$.

Moreover, the subset of $\mathcal{H}$ ilb $_{p(z)}^{n}$ corresponding to $\mathcal{S} t_{h}\left(\dot{\mathfrak{j}}_{\geqslant r}\right)$ always contains the one corresponding to $\mathcal{S} t_{h}(j)$ and the inclusion can be strict, because points in $\mathcal{S} t_{h}(\mathfrak{j})$ correspond to ideals defining subschemes in $\boldsymbol{P}^{n}$ with the same Hilbert function as the subscheme $Z=\mathcal{V}(\mathfrak{j})$, while being in $\mathcal{S} t_{h}\left(\dot{j}_{\geqslant r}\right)$ only requires the same Hilbert polynomial. An interesting example of this type is that of the lexicographic saturated ideal $\mathbb{R}$ such that $k\left[X_{0}, \ldots, X_{n}\right] / \mathcal{Q}$ has Hilbert polynomial $p(z)$ and whose regularity is indeed the Gotzmann number $r$ of $p(z)$ : in $\S 7$ we show that $\mathcal{S} t_{h}\left(\Omega_{\geqslant r}\right)$ is isomorphic to an open subset of the Reeves and Stillman component $H_{R S}$ of $\mathcal{H}$ illb ${ }_{p(z)}^{n}$, while in general $\mathcal{S t}_{h}(\mathfrak{\Omega})$ corresponds to a locally closed subscheme of lower dimension (see [22, Remark 4.8]). 
The main reason of our setting is contained in Theorem 6.3. Let $p(z)$ be any admissible Hilbert polynomial in $\boldsymbol{P}^{n}$ with Gotzmann number $r$ and let $\prec$ be a fixed term ordering on monomials of $k\left[X_{0}, \ldots, X_{n}\right]$. Following the classical construction, we consider $\mathcal{H i l b}_{p(z)}^{n}$ as a subscheme of a projective space through the Plücker embedding of the grassmannian $\mathrm{Gr}(t, M)$, where $M=\operatorname{dim}_{k}\left(k\left[X_{0}, \ldots, X_{n}\right]_{r}\right)$ and $t=M-p(r)$. The simple remark that the Plücker coordinates correspond to sets of $t$ distinct monomials of degree $r$ (that we can write in decreasing order with respect to $\prec$ ), allows us to get a lexicographic total order on them. If $j_{0}$ is a monomial ideal generated in degree $r$ such that $k\left[X_{0}, \ldots, X_{n}\right] / \dot{j}_{0}$ has Hilbert polynomial $p(z)$ with Gotzmann number $r$, then we show that $\mathcal{S} t_{h}\left(\dot{j}_{0}\right)$ is the locally closed subscheme of $\mathcal{H i l b} b_{p(z)}^{n}$ given by the conditions that the Plücker coordinate corresponding to the monomial basis of $\dot{j}_{0}$ does not vanish and the bigger ones vanish.

As a consequence we are able to prove that every irreducible and reduced component of $\mathcal{H i l b}_{p(z)}^{n}$ (or of its support) has an open subset which is a homogeneous affine variety (with respect to a non-standard grading). Especially, if $j$ is generated by the $t$ largest degree $r$ monomials (we call it a $(r, \prec)$-segment ideal), then $\mathcal{S t}_{h}(\mathrm{j})$ is naturally isomorphic to an open subset of $\mathcal{H i l b}_{p(z)}^{n}$. Therefore we can easily deduce a few interesting properties of rationality for the components of Hilbert schemes (see Theorem 6.3 iii), Corollary 6.9, Corollary 6.10, Corollary 7.1):

Theorem C. Let $H$ be an irreducible component of $\mathcal{H}$ ilb $b_{p(z)}^{n}$.

- If $H$ is smooth, then it is rational. The same holds for its support Supp $H$.

- If H contains a smooth point which corresponds to a $(r, \prec)$-segment ideal (where $\prec$ is any term ordering), then $H$ is rational. The same holds for $\operatorname{Supp} H$.

- The Reeves and Stillman component $H_{R S}$ of $\mathcal{H} \mathrm{ilb}_{p(z)}^{n}$ is rational.

The last item can be obtained as a direct consequence of the previous one, because the lexicographic saturated ideal $\mathbb{2}$ corresponds to a smooth point in $H_{R S}$, as proved by Reeves and Stillman in [20], and $\mathbb{L}_{\geqslant r}$ is a $(r$, Lex)-segment ideal. However, we can also get a new proof of this fact, not applying the quoted result by Reeves and Stillman, but proving that $\mathcal{S t}_{h}\left(\Omega_{\geqslant r}\right)$ is isomorphic to an affine space using our method based on the minimal embedding (see Theorem 7.3). 
In $\S 8$ we present a pseudo-code description of the procedures based on our results, that can be implemented using one of the several softwares for symbolic computation. With such a procedure we are able to write equations for some Gröbner strata corresponding to the Hilbert scheme $\mathcal{H i l b}_{4 z}^{3}$. In this way we find a computational confirmation of the results obtained by Gotzmann in [9], namely that $\mathcal{H i l b}_{4 z}^{3}$ has two components of dimensions 23 and 16 respectively and also some improvements. In fact, we also show that both components of that Hilbert scheme are rational (because each of them contains a smooth point corresponding to a segment ideal), they have transversal intersection (studied using a third segment ideal) and finally that the forth segment ideal allowed by the Hilbert polynomial $p(z)=4 z$ is singular point whose stratum has dimension 23 and embedding dimension 27.

The paper is organized as follows. $\S 2$ contains some general notation.

In $\S 3$, we take up the construction of Gröbner strata made in [22] and prove that they are well defined (Theorem 3.6).

In $\S 4$ we discuss the main properties of Gröbner strata as homogeneous varieties with respect to a non-standard grading and we obtain some useful criterion in order to know when Borel-fixed monomial ideals with the same saturation define the same Gröbner stratum (Theorem 4.7).

In $\S 5$, we focus our attention on ideals generated in degree $r$, where $r$ is the Gotzmann number of their Hilbert polynomials, and prove that their Gröbner strata can be defined by minors of suitable matrices (Proposition 5.5).

$\S 6$ represents the heart of the work. We show that there is a close connection between the above quoted matrices defining Gröbner strata and those appearing in the classical construction of Hilbert schemes and obtain as a consequence the main results of the paper about rational components (Theorem 6.3 iii)).

Finally, in $\S 7$, we prove the rationality of the Reeves-Stillman component $H_{R S}$ of $\mathcal{H}$ ilb $b_{p(z)}^{n}$ using our method, based on the minimal embedding and in $\S 8$ we apply this same method in order to perform some explicit computations about $\mathcal{H}$ ilb $b_{4 z}^{3}$.

\section{Notation.}

Throughout the paper, we will consider the following general notation.

1. During the construction of Gröbner strata, we work on a field $k$ of any characteristic, whereas when we study the Hilbert scheme, we will suppose that $k$ is algebraically closed. 
2. $k\left[X_{0}, \ldots, X_{n}\right]$ is the polynomial ring in the set of variables $X_{0}, \ldots, X_{n}$ that we will often denote by the compact notation $X$, so that $k[X]:=k\left[X_{0}, \ldots, X_{n}\right]$; we will denote by $X^{\alpha}$ the generic monomial in $k[X]$, where $\alpha$ represents a multi-index $\left(\alpha_{0}, \ldots, \alpha_{n}\right)$, that is $X^{\alpha}:=X_{0}^{\alpha_{0}} \cdots X_{n}^{\alpha_{n}}$. j will be a monomial ideal in $k[X]$ with basis $\left\{X^{\gamma_{1}}, \ldots, X^{\gamma_{t}}\right\}$ and $\operatorname{Syz}(\mathfrak{j})$ its $k[X]$-module of syzygies.

$X^{\alpha} \mid X^{\gamma}$ means that $X^{\alpha}$ divides $X^{\gamma}$, that is there exists a monomial $X^{\beta}$ such that $X^{\alpha} \cdot X^{\beta}=X^{\gamma}$. If such monomial does not exist, we will write $X^{\alpha} \nmid X^{\gamma}$.

$\prec$ will be a fixed term ordering on the set ${ }^{\top} T_{X}$ of monomials in $k[X]$ and we always assume that $X_{0} \succ \ldots \succ X_{n}$. As the term order $\prec$ is fixed, we often omit to indicate it. Given a monomial $X^{\alpha}$, we refer with $\min \left(X^{\alpha}\right)$ as the smallest variable dividing the monomial, that is $\min \left(X^{\alpha}\right)=$ $\min \left\{X_{i}\right.$ s.t. $\left.X_{i} \mid X^{\alpha}\right\}$. We will denote also by $\prec$ its extension to the multiplicative group of Laurent monomials $\bar{T}_{X}$ and the corresponding total ordering on $Z^{n+1}$ given by $\alpha \prec \beta \Leftrightarrow X^{\alpha} \prec X^{\beta}$.

For every polynomial $F$ in $k[X]$ (or $k[X, C], k[C]$ ), $\operatorname{LT}(F)$ is its leading term with respect to the fixed term ordering; in the same way, if $\mathfrak{a}$ is an ideal, $\operatorname{LT}(\mathfrak{a})$ is its initial ideal.

3. We will introduce a second set of variables $C_{i \alpha}$ that we will denote with $C$. So $k[X, C]$ will be the polynomial ring in the variables $X$ and $C$ and ${ }^{\top} T_{X, C}$ the corresponding set of monomials. The term ordering on ${ }^{\prime} T_{X, C}$ will be induced by the term ordering on ${ }^{~} \mathrm{~T}_{X}$ and it will be an elimination ordering of the variables $X$ that will coincide with $\prec$ on ${ }^{\top} T_{X}$ : so we will denote by the same symbol $\prec$ also this term orderings on ${ }^{~} T_{X, C}$ and its restriction to ${ }^{~} T_{C}$.

4. Let $G$ be any polynomial in $k[C, X]$. An $X$-monomial of $G$ is a monomial of $T_{X}$ that appears in $G$ considered as a polynomial in the variables $X$ with coefficients in the ring $k[C]$; the $X$-coefficients of $G$ are the elements of $k[C]$ that are coefficients of an $X$-monomial. Note that the $X$ coefficients are polynomials, but not necessary monomials.

5. Given any subscheme $Z$ in $\boldsymbol{P}^{n}$, we will denote by $\operatorname{Supp} Z$ its support and by $I(Z)$ the saturated ideal in $k[X]$ that defines $Z$. Given any ideal $\mathfrak{a}$, we will denote by $\mathcal{V}(\mathfrak{a})$ the affine scheme $\operatorname{Spec}(k[X] / \mathfrak{a})$.

6. $\mathcal{H}$ ilb $b_{p(z)}^{n}$ will denote the Hilbert scheme parameterizing all subschemes $Z$ in $\boldsymbol{P}^{n}$ with Hilbert polynomial $p(z) . r$ will be the Gotzmann number of $p(z)$, that is the worst Castelnuovo-Mumford regularity among subschemes parameterized by $\mathcal{H}$ ill $b_{p(z)}^{n}$. When we write that an ideal $\dot{i} \subset k[X]$ belongs to $\mathcal{H}$ ill $b_{p(z)}^{n}$, we will mean that $i$ is generated in degree $r$ and that the Hilbert polynomial of $\operatorname{Proj} k[X] / \mathfrak{i}$ is $p(z)$. By abuse of notation we will say that any such ideal $i$ has Hilbert polynomial $p(z)$ referring to the 
Hilbert polynomial of the quotient, even if the real Hilbert polynomial of $i$ is $\left(\begin{array}{c}z+n \\ n\end{array}\right)-p(z)$.

\section{The ideal of a Gröbner Stratum.}

Now we introduce the Gröbner strata and prove some properties, generalizing definitions and results of the paper [22].

Definition 3.1. The tail of $X^{\gamma}$ with respect to $j$ (and to the fixed term ordering $\prec$ ) is the set of monomials:

$$
T_{\gamma}^{\prec}=\left\{X^{\alpha} \in{ }^{\top} \mathbb{T}_{X} \mid X^{\alpha} \prec X^{\gamma}, X^{\alpha} \notin j\right\}
$$

Every ideal $\mathfrak{i}$ such that $\mathrm{LT}(\mathfrak{i})=\mathfrak{j}=\left(X^{\gamma_{1}}, \ldots, X^{\gamma_{t}}\right)$ has a reduced Gröbner basis of the type $\left\{f_{1}, \ldots, f_{t}\right\}$ where:

$$
f_{i}=X^{\gamma_{i}}+\sum_{X^{\alpha} \in T_{\gamma_{i}}^{\prec}} c_{i \alpha} X^{\alpha}
$$

and $c_{i \alpha} \in k, c_{i \alpha}=0$ except finitely many of them. It is very natural to parameterize the family of all the ideals $i$ by the coefficients $c_{i \alpha}$; in this way it corresponds to a subset of $k^{T^{\prec}}$, where $T^{\prec}=T_{\gamma_{1}}^{\prec} \times \cdots \times T_{\gamma_{t}}^{\prec}$.

In many interesting cases, $T_{\gamma_{i}}^{\prec}$ are finite sets and so $k^{T^{\prec}}$ is an affine space: this happens for instance if $j$ is a zero-dimensional ideal or if $\prec$ is a suitable term ordering; in other cases, for instance when only homogeneous ideals are concerned, $T^{\prec}$ can be infinite, but we can restrict our interest to a suitable finite subset. The following definition extends and includes all the previous cases.

Definition 3.2. Let us fix $T=\left\{T_{1}, \ldots, T_{t}\right\}$ where $T_{i}$ is a finite subset of the tail of $X^{\gamma_{i}}$ with respect to $\dot{j}$. We will denote by $\mathcal{S} t(\dot{\mathfrak{j}}, T)$ the family of all ideals $\mathfrak{i}$ in $k[X]$ such that $\operatorname{LT}(\mathfrak{i})=j$ and whose reduced Gröbner basis $f_{1}, \ldots, f_{t}$ is of the type:

$$
f_{i}=X^{\gamma_{i}}+\sum_{X^{\alpha} \in T_{i}} c_{i \alpha} X^{\alpha} .
$$

Moreover we will use the following special notation:

i) $\mathcal{S t}(\mathrm{j})$, if $T_{i}=T_{\gamma_{i}}^{\prec}$ (of course only if $T_{\gamma_{i}}^{\prec}$ are finite sets): $\mathcal{S t}(\mathrm{j})$ parameterizes all the ideals $\dot{i}$ such that $\operatorname{LT}(\dot{i})=\dot{j}$.

ii) $\mathcal{S} t_{h}(\mathrm{j})$, if $T_{i}$ is the subset of $T_{\gamma_{i}}^{\prec}$ of the monomials with the same degree as $X^{\gamma_{i}}: \mathcal{S} t_{h}(\mathfrak{j})$ parameterizes all the homogeneous ideals $\mathfrak{i}$ such that $\operatorname{LT}(\mathfrak{i})=j$. 
REMARK 3.3. It will be clear later that the term ordering affects the construction of a Gröbner stratum only because it states which monomials can belong to the tails; in fact two different term orderings giving the same tails will lead to the same Gröbner strata.

Every ideal $\mathfrak{i}$ in the family $\mathcal{S} t(\mathfrak{j}, T)$ is uniquely determined by a point in the affine space $\mathrm{A}^{N}\left(N=\sum_{i}\left|T_{i}\right|\right)$ where we fix coordinates $C_{i \alpha}$ corresponding to the coefficients $c_{i \alpha}$ that appear in (3.3). The subset of $\mathrm{A}^{N}$ corresponding to $\mathcal{S} t(\mathfrak{j}, T)$ turns out to be a closed algebraic set. More precisely, we will see how it can be endowed in a very natural way with a structure of affine subscheme, possibly reducible or non reduced, that is we will see that it can be obtained as the subscheme of $\mathrm{A}^{N}$ defined by an ideal $\mathfrak{h}(\mathfrak{j}, T)$ in $k[C]$, where $C$ is the set of variables $C_{i \alpha}$.

In the following, we refer to the terminology introduced in Notation 4 for what concerns the polynomials in $k[X, C]$.

Definition 3.4. We will denote by $\mathfrak{h}(\mathfrak{j}, T)$ and $L(\mathfrak{j}, T)$ respectively any ideal in $k[C]$ that can be obtained in the following way.

- Let $\mathcal{B}=\left\{F_{1}, \ldots, F_{t}\right\}$ be the set of polynomials in $k[X, C]$ given by:

$$
F_{i}=X^{\gamma_{i}}+\sum_{X^{\alpha} \in T_{i}} C_{i \alpha} X^{\alpha} .
$$

- Consider any term order in $k[X, C]$ which is an elimination order for the variables $X$ and that coincides with $\prec$ for monomials in ${ }^{T}{ }_{X}$; there will be no confusion if we denote it by the same symbol $\prec$. With respect to such a term order, the leading term of $F_{i}$ is $X^{\gamma_{i}}$.

- Fix the subset $P$ of $\{(i, j) \mid 1 \leqslant i<j \leqslant m\}$ corresponding to any set of generators for $\mathrm{Syz}(\mathrm{j})$;

- For every $(i, j) \in P$, let $R_{i j}$ be a complete reduction of the $S$-polynomial $S\left(F_{i}, F_{j}\right)$ with respect to $\mathcal{B}$.

- For every $(i, j) \in P$, let $M_{i j}$ be a complete reduction of $S\left(F_{i}, F_{j}\right)$ with respect to $j$.

- $\mathfrak{h}(\mathfrak{j}, T)$ is the ideal in $k[C]$ generated by the $X$-coefficients of the polynomials $R_{i j},(i, j) \in P$.

- $L(\mathrm{j}, T)$ is the $k$-vector space in $\langle C\rangle$ generated by the $X$-coefficients of $M_{i j},(i, j) \in P$.

It is almost evident, that the definition of $\mathfrak{h}(\mathfrak{j}, T)$ is nothing else than Buchberger's characterization of Gröbner basis if we think to the $C_{i \alpha}$ 's as constant in $k$ instead of variables. In fact the variables $C$ do not appear in 
the leading terms of $F_{i}$ and so their specialization in $k$ commutes with reduction with respect to $\mathcal{B}$. Thus $\left(\ldots, c_{i \alpha}, \ldots\right)$ is a closed point in the support of $\mathcal{V}(\mathfrak{G}(\mathfrak{j}, T))$ in $\mathrm{A}^{N}$ if and only if it corresponds to polynomials $f_{1}, \ldots, f_{m}$ in $k[X]$ that are a Gröbner basis. Then the support of $\mathcal{V}(\mathfrak{h}(\mathfrak{j}, T))$ is uniquely defined; however a priori the ideal $\mathfrak{h}(\mathfrak{i}, T)$ could depend on the choices we perform computing it, that is on the choice of the set $P$ of generators for $\operatorname{Syz}(\mathfrak{j})$ and on the choice of a reduction for the $S$-polynomials $S\left(F_{i}, F_{j}\right)$ with respect to $\mathcal{B}$ (which in general is not uniquely determined).

Thanks again to Buchberger's criterion, we can prove that in fact $\mathfrak{h}(\mathfrak{j}, T)$ only depends on $\dot{j}, T$ and of course $\prec$ because it can be defined in an equivalent intrinsic way.

Proposition 3.5. Let $j \subseteq k[X], \mathcal{B}=\left\{F_{1}, \ldots, F_{t}\right\} \subset k[X, C]$ and $\prec$ be as above and consider an ideal a in $k[C]$ with Gröbner basis $\mathcal{A}$. The following are equivalent:

i) $\mathcal{B} \cup \mathcal{A}$ is a Gröbner basis in $k[X, C]$;

ii) a contains the $X$-coefficients of all the polynomials in the ideal $\left(F_{1}, \ldots, F_{t}\right) k[X, C]$ that are reduced modulo $;$;

iii) a contains all the $X$-coefficients of every complete reduction of $S\left(F_{i}, F_{j}\right)$ with respect to $\mathcal{B}$ for every $i, j$;

iv) a contains all the $X$-coefficients of some (even partial) reduction with respect to $\mathcal{B}$ of $S\left(F_{i}, F_{j}\right)$ for every $i, j$;

$v)$ a contains all the $X$-coefficients of some (even partial) reduction with respect to $\mathcal{B}$ of $S\left(F_{i}, F_{j}\right)$, for every $(i, j)$ corresponding to a set of generators of $\operatorname{Syz}(\mathfrak{j})$.

Proof. $i) \Rightarrow i i)$ : let $G$ a polynomial in $\left(F_{1}, \ldots, F_{t}\right) k[X, C]$ which is reduced modulo j. By hypothesis, $G$ must be reducible to 0 through $\mathcal{B} \cup \mathcal{A}$, so that the next step of reduction have to be performed just using $\mathcal{A}$. But any step of reduction through $\mathcal{A}$ does not change the $X$-monomials and only modifies the $X$-coefficients; then $G \stackrel{\mathcal{A}}{\longrightarrow} 0$, that is every $X$-coefficient in $G$ can be reduced to 0 using $\mathcal{A}$ : this shows that all the $X$-coefficients in $G$ belong to $a$.

$i i) \Rightarrow i i i), i i i) \Rightarrow i v)$ and $(v) \Rightarrow v$ ) are obvious.

$v) \Rightarrow i$ ): we can check that $\mathcal{B} \cup \mathcal{A}$ is a Gröbner basis using the refined Buchberger criterion (see for instance [4, Theorem 9, pag. 104]). If $\mathcal{A}=\left\{a_{1}, \ldots, a_{r}\right\}$, a set of generators for $\operatorname{Syz}\left(X^{\gamma_{1}}, \ldots, X^{\gamma_{t}}, \operatorname{LT}\left(a_{1}\right), \ldots, \operatorname{LT}\left(a_{r}\right)\right)$ can be obtained as the union of a set of generators for $\operatorname{Syz}\left(X^{\gamma_{1}}, \ldots, X^{\gamma_{t}}\right)$, a set of generators for $\operatorname{Syz}\left(\operatorname{LT}\left(a_{1}\right), \ldots, \operatorname{LT}\left(a_{r}\right)\right)$ and the obvious syzygies of $\left(X^{\gamma_{i}}, \operatorname{LT}\left(a_{j}\right)\right)$. Then: 
- $S\left(a_{i}, a_{j}\right) \stackrel{\mathcal{B} \cup \mathcal{A}}{\longrightarrow} 0$, since $\mathcal{A}$ is a Gröbner basis and $\mathcal{A} \subseteq \mathcal{B} \cup \mathcal{A}$;

- $S\left(a_{i}, F_{j}\right) \stackrel{\mathcal{B} \cup \mathcal{A}}{\longrightarrow} 0$, since the leading terms of $a_{i}$ and $F_{j}$ are coprime and $a_{i}, F_{j} \in \mathcal{B} \cup \mathcal{A}$;

- $S\left(F_{i}, F_{j}\right) \stackrel{\mathcal{B} \cup \mathcal{A}}{\longrightarrow} 0$ in at least one way, by hypothesis.

There are many ideals a fulfilling the equivalent conditions of Proposition 3.5: for instance we can consider the irrelevant maximal ideal in $k[C]$ or any ideal obtained accordingly with condition iv). Moreover, if a satisfies those conditions and $\mathfrak{a}^{\prime} \supset \mathfrak{a}$, then also $\mathfrak{a}^{\prime}$ does, and if the ideals $\mathfrak{a}_{l}$ satisfy the conditions, then also their intersection $\bigcap a_{l}$ does. As a consequence of these remarks we obtain the proof of the uniqueness of the ideal $\mathfrak{h}(\mathfrak{j}, T)$ given by Definition 3.4.

THEOREM 3.6. Let $j$ and $T$ as above. Then:

i) $\mathfrak{h}(\mathfrak{j}, T)$ is uniquely defined; in fact $\mathfrak{h}(\mathfrak{j}, T)=\bigcap \mathfrak{a}$, a satisfying the equivalent conditions of Proposition 3.5.

ii) $L(\dot{\mathfrak{j}}, T)$ is uniquely defined.

Proof. $i$ ): $\mathfrak{h}$ is one of the ideals $\mathfrak{a}$, because it satisfies condition $v$ ); on the other hand, if a satisfies condition $i i i)$, then clearly $\mathfrak{a} \supseteq \mathfrak{h}$.

For $i i)$ it is sufficient to observe that the generators for $L(\dot{\mathfrak{i}}, T)$ are the degree 1 homogeneous components (here "homogeneous" is related to the usual grading of $k[C]$ that is the Z-grading with variables of degree 1) of the generators of $\mathfrak{h}(\mathfrak{i}, T)$ given in its construction (Definition 3.4).

By abuse of notation we will denote by the same symbol $\mathcal{S} t(\mathrm{j}, T)$ the family of ideals and the subscheme in $\mathrm{A}^{N}$ given by the ideal $\mathfrak{h}(\mathfrak{i}, T)$. Note that $\mathfrak{h}(\mathfrak{i}, T)$ is not always a prime ideal and so $\mathcal{S} t(\mathfrak{j}, T)$ is not necessarily irreducible nor reduced, as the following trivial example shows.

ExAMPLE 3.7. Let $j=\left(x^{2}, x y\right) \subset k[x, y]$ and $\prec$ be any term ordering. Let us choose $T=\left\{T_{x^{2}}=\emptyset, T_{x y}=\{y\}\right\}$ and construct the ideal of the Gröbner stratum $\mathcal{S t}(\dot{\mathfrak{j}}, T)$ according to Definition 3.4:

$$
\begin{gathered}
\left\{F_{1}=x^{2}, F_{2}=x y+C y\right\}, \\
S_{12}=y F_{1}-x F_{2}=-C x y \stackrel{\left\{F_{1}, F_{2}\right\}}{\longrightarrow} R_{12}=-C x y+C F_{2}=C^{2} y .
\end{gathered}
$$

Then $\mathfrak{h}(\mathfrak{j}, T)=\left(C^{2}\right)$ that is $\mathcal{S} t(\mathfrak{j}, T)$ is a double point in the affine space $\mathbb{A}^{1}$. 


\section{Gröbner strata are homogeneous varieties.}

In this section we will see how every Gröbner stratum $\mathcal{S} t(\mathfrak{j}, T)$ is in a very natural way homogeneous with respect to a suitable non-standard grading on $k[C]$, so that we can apply the nice properties typical of this kind of schemes and especially those obtained in [22] and in [8].

For the meaning of $j, k[X],\left\{X^{\gamma_{1}}, \ldots, X^{\gamma_{t}}\right\}$ and $\prec$ we refer to Notation 2 and for $k[X, C],\left\{F_{1}, \ldots, F_{t}\right\}, \mathcal{S} t(\mathfrak{j}, T), \mathfrak{h}(\mathfrak{i}, T)$ to the previous section.

First of all, we recall the definitions and properties that we will use more often.

Definition 4.1. We will consider $k[X, C]$ and $k[C]$ as graded ring over the totally ordered group $\left(Z^{n+1},+, \prec\right)$ with grading $\lambda$ given by $\lambda\left(X^{\alpha}\right)=\alpha$ and $\lambda\left(C_{i \alpha}\right)=\gamma_{i}-\alpha$.

As we will use also the usual grading over $Z$ where all the variables have degree 1 , we will always write explicitly the symbol $\lambda$ when the above defined grading is concerned (so, $\lambda$-degree $l$ with $l \in Z^{n+1}, \lambda$ homogeneous of degree $l$ etc.) and leave the simple terms when the usual grading is involved (so, degree $r$ with $r \in Z$, homogeneous of degree $r$ etc.).

Proposition 4.2. (See [22, Lemma 2.8])

i) The grading $\lambda$ is positive.

ii) $\mathfrak{h}(\mathfrak{j}, T)$ is a $\lambda$-homogeneous ideal.

Proof. i) Let us observe that all the variables have $\lambda$-degree higher than that of the constant 1 . In fact $\lambda\left(X_{i}\right) \succ \lambda(1)$ because $\prec$ is a term ordering and $\lambda\left(C_{i \alpha}\right) \succ \lambda(1)$ because, $X^{\gamma_{i}} \succ X^{\alpha}$ by definition of tails. As well known, this condition is equivalent to the positivity of the grading (see [15, Chapter 4]).

ii) We observe that $\lambda$ on $\bar{T}_{C}$ is the restriction of the grading on $\bar{T}_{X, C}$. Every monomial that appears in $F_{i}$ is of the type $C_{i \alpha} X^{\alpha}$ and so its $\lambda$-degree is $\lambda\left(C_{i \alpha} X^{\alpha}\right)=\lambda\left(C_{i \alpha}\right)+\lambda\left(X^{\alpha}\right)=\gamma_{i}$. Thus all the polynomials $F_{i}$ are $\lambda$-homogeneous and then also the $S$-polynomials $S\left(F_{i}, F_{j}\right)$ and their reductions are $\lambda$-homogeneous. Finally, the $X$-coefficients in any $\lambda$-homogeneous polynomial (which are polynomials in $k[C]$ ) are $\lambda$-homogeneous.

We now recall some properties of $L(\mathfrak{j}, T)$ (see also [22, Proposition 2.4] and [8, Theorem 3.2]). 
Proposition 4.3. The linear space $\mathcal{V}(L(\mathfrak{j}, T))$ can be naturally identified with the Zariski tangent space to $\mathcal{S} t(\mathrm{j}, T)$ at the origin.

If $C^{\prime \prime} \subset C$ is any subset of ed $:=\operatorname{dim} \mathcal{V}(L(\mathfrak{j}, T))$ variables such that $L(\mathfrak{j}, T) \oplus\left\langle C^{\prime \prime}\right\rangle=\langle C\rangle$, then $\mathfrak{h}(\mathfrak{j}, T) \cap k\left[C^{\prime \prime}\right]$ defines a $\lambda$-homogeneous subvariety in $\mathrm{A}^{\text {ed }}$ isomorphic to $\mathcal{S} t(\mathfrak{h}, T)$.

We may summarize the previous result saying that $\mathcal{S} t(\mathfrak{h}, T)$ can be embedded in its Zariski tangent space at the origin. This explains the following terminology.

DeFinition 4.4. The number ed is the embedding dimension of $\mathcal{S} t(\mathfrak{j}, T)$. The complement $C^{\prime}:=C \backslash C^{\prime \prime}$ is a maximal set of eliminable variables for $\mathfrak{h}(\mathfrak{j}, T)$.

COROLLARY 4.5. In the above notation, the following statements are equivalent:

1. $\mathcal{S} t(\mathrm{j}, T) \simeq \mathrm{A}^{\mathrm{ed}}$;

2. $\mathcal{S} t(\mathfrak{j}, T)$ is smooth;

3. the origin is a smooth point for $\mathcal{S} t(\mathfrak{j}, T)$;

4. ed $\leqslant \operatorname{dim} \mathcal{S} t(\mathfrak{j}, T)$.

Note that in general a maximal set of eliminable variables (and so its complementary) is not uniquely determined. However, if $C_{i \alpha} \in L(\mathrm{j}, T)$, then $C_{i \alpha}$ belongs to any set of eliminable variables; on the other hand, if $C_{i \alpha}$ does not appear in any element of $L(\mathfrak{j}, T)$, then $C_{i \alpha}$ does not belong to any set of eliminable variables.

There is an easy criterion that allows us to decide if a variable is eliminable or not.

CRITERION 4.6. Let $\operatorname{LT}\left(F_{i}\right)=X^{\gamma_{i}}, \operatorname{LT}\left(F_{j}\right)=X^{\gamma_{j}}$ and let $C_{i \beta}$ be a variable appearing in the tail of $F_{i}$. Using the reduction with respect to $j$ of a $\lambda$ homogeneous polynomial $X^{\delta} F_{i}-X^{\eta} F_{j}$ we can see that:

i) if $X^{\delta+\beta} \notin j$ and $X^{\delta+\beta-\eta}$ is not a monomial that appears in $F_{j}$, then $C_{i \beta} \in L(\mathfrak{j}, T)$;

ii) if $X^{\delta+\beta} \notin j$ and $X^{\beta^{\prime}}=X^{\delta+\beta-\eta}$ is a monomial that appears in $F_{j}$, then $C_{i \beta}-C_{j \beta^{\prime}} \in L(\mathfrak{j}, T)$

Moreover if $C_{i \beta}-C_{j \beta^{\prime}} \in L(\mathfrak{j}, T)$, then every maximal set of eliminable variables must contain at least either one of them. 
In most cases the number $N=|C|$ is very big and $\mathfrak{h}(\mathfrak{j}, T)$ needs a lot of generators so that finding it explicitly is a very heavy computation. On the contrary $L(j, T)$ is very fast to compute and so we can easily obtain a set of eliminable variables $C^{\prime}$; a forgoing knowledge of $C^{\prime}$ allows a simpler computation of the ideal $\mathfrak{h}(\mathfrak{j}, T) \cap k\left[C \backslash C^{\prime}\right]$ that gives $\mathcal{S} t(\mathfrak{j}, T)$ embedded in the affine space of minimal dimension $\mathrm{A}^{\text {ed }}$.

Furthermore, in many interesting cases we can greatly bring down the number of involved variables thanks to another kind of argument.

THEOREM 4.7. Let $\dot{j} \subset k\left[X_{0}, \ldots, X_{n}\right]$ be a Borel-fixed saturated monomial ideal with basis $B, m$ any integer and $\mathfrak{h}_{m}:=\mathfrak{h}\left(\mathfrak{j}_{\geqslant m}\right)$ the ideal of $\mathcal{S} t_{h}\left(\mathfrak{j}_{\geqslant m}\right)$ as in Definition 3.4.

i) There is a set of eliminable variables for $\mathfrak{h}_{m}$ that contains all variables except at most the ones appearing in polynomials $F_{i}$ whose leading term is either $X^{\gamma} \in B_{\geq m}$ or $X^{\alpha} X_{n}^{m-|\alpha|}$, where $X^{\alpha} \in B_{<m}$.

ii) $\mathcal{S} t_{h}\left(\dot{\mathrm{j}}_{\geqslant m-1}\right)$ is a closed subscheme of $\mathcal{S} t_{h}\left(\dot{\mathrm{i}}_{\geqslant m}\right)$. More precisely $\mathcal{S} t_{h}\left(\dot{\mathrm{j}}_{\geqslant m-1}\right) \simeq \mathcal{S} t_{h}\left(\dot{\mathrm{j}}_{\geqslant m}, T\right)$ where $T$ contains the complete tail of a monomial in the basis of $\dot{j}_{\geqslant m}$ if it is not divided by $X_{n}$, and a tail containing only monomials divided by $X_{n}$ otherwise.

iii) If $X_{n-1}$ does not appear in any monomial of degree $m+1$ in the monomial basis of $\mathfrak{j}$, then $\mathcal{S} t_{h}\left(\dot{\mathrm{j}}_{\geqslant m-1}\right) \simeq \mathcal{S} t_{h}\left(\dot{\mathrm{j}}_{\geqslant m}\right)$.

iv) If $X_{n-1}$ appears in $N$ monomials of degree $m+1$ in the monomial basis of $\dot{j}$, then ed $\mathcal{S} t_{h}\left(\dot{j}_{\geqslant m}\right) \geqslant \operatorname{ed} \mathcal{S} t_{h}\left(\dot{j}_{\geqslant m-1}\right)+N M$, where $M$ is the number of monomials of the basis of $j$ of degree smaller than $m$.

v) $\mathcal{S} t_{h}\left(\dot{\mathfrak{j}}_{\geqslant m-1}\right) \not \mathcal{S} t_{h}\left(\dot{\mathfrak{j}}_{\geqslant m}\right)$ if and only if $X_{n-1}$ appears in monomials of degree $m+1$ in the monomial basis of $\dot{j}$ and $\dot{j}_{\geqslant m-1} \neq \dot{j} \geqslant m$.

vi) If $s$ is the maximal degree of a monomial divided by $X_{n-1}$ in the monomial basis of $\mathfrak{j}$, then $\mathcal{S} t_{h}\left(\mathfrak{j}_{\geqslant s-1}\right) \simeq \mathcal{S} t_{h}\left(\mathfrak{j}_{\geqslant m}\right)$ for every $m \geqslant s$.

Proof. i) Let us consider any monomial $X^{\eta}$ in the monomial basis of $\dot{j}_{\geqslant m}$ which does not belong to $B \geqslant m$ and such it that could be written as $X^{\eta}=X^{\alpha} X^{\varepsilon}$ where $X^{\alpha}$ is a minimal generator of $j$ of degree $d<m$ and $X^{\varepsilon}$ is a monomial of degree $m-d, X^{\varepsilon} \neq X_{n}^{m-d}$. Then among the polynomials $F_{i}$ there are:

$$
\begin{aligned}
& F=X^{\alpha} X_{n}^{m-d}+\sum C_{\beta} X^{\beta}, \\
& F^{\prime}=X^{\alpha+\varepsilon}+\sum C_{\delta}^{\prime} X^{\delta} .
\end{aligned}
$$

We have to prove that all the variables $C^{\prime}$ that appear in $F^{\prime}$ can be eliminated. 
The $S$-polynomial of $F$ and $F^{\prime}$ is:

$$
S\left(F, F^{\prime}\right)=X_{n}^{p} F^{\prime}-X^{\varepsilon^{\prime}} F=\sum C_{\delta}^{\prime} X^{\delta} X_{n}^{p}-\sum C_{\beta} X^{\beta+\varepsilon^{\prime}} .
$$

No monomial $X^{\delta} X_{n}^{p}$ in the first summand belongs to $\dot{\mathfrak{l}}_{\geqslant m}$ because $X^{\delta} \notin \dot{j}$ and $\dot{j}$ is saturated and Borel-fixed. Thus, the linear part of the coefficient of $X^{\delta} X_{n}^{p}$ in the reduction of this $S$-polynomial will be either $C_{\delta}^{\prime}$ or $C_{\delta}^{\prime}-C_{\beta}$. Then $C^{\prime}$ is a set of eliminable variables for $\dot{j}_{\geqslant m}$.

ii) The first part of this statement is a special case of general facts proved in $[11, \S 3]$.

We directly prove the second part (which implies the first one). Here we denote by $X^{\alpha}$ and $X^{\gamma}$ the monomials in the basis of $\dot{j}_{\geqslant m-1}$ of degree $m-1$ and $\geq m$ respectively, and we set:

$$
\begin{aligned}
G_{\alpha} & :=X^{\alpha}+\sum C_{\alpha \delta} X^{\delta} \\
G_{\gamma} & :=X^{\gamma}+\sum C_{\gamma \eta} X^{\eta}
\end{aligned}
$$

where $X^{\delta}$ varies among all monomials of degree $m-1$ in the tail of $X^{\alpha}$ and $X^{\eta}$ among those of the same degree as $X^{\gamma}$ in its tail. Applying the procedure described in Definition 3.4 on the set of polynomials $G$ we define $\mathcal{S} t_{h}\left(\mathfrak{j}_{\geqslant m-1}\right)$ by an ideal $\overline{\mathfrak{h}} \subset k[C]$.

The basis of $\dot{j}_{\geqslant m}$ is made by monomials of the following three types:

- monomials $X^{\gamma}$ of degree $\geq m$, that also belong to the basis of $j_{\geq m-1}$;

- monomials $X^{\alpha} X_{n}$ such that $X^{\alpha}$ is any monomial of degree $m-1$ in the basis of $\dot{j}_{\geqslant m-1}$;

- monomials $X^{\alpha} X_{i}$ of degree $m$ such that $X^{\alpha}$ is as above and $\min \left(X^{\alpha}\right) \geq X_{i} \neq X_{n}$

We set:

$$
\begin{aligned}
F_{\alpha n} & :=X^{\alpha} X_{n}+\sum C_{\alpha \delta} X^{\delta} X_{n} \\
F_{\alpha i} & :=X^{\alpha} X_{i}+\sum C_{\alpha i \tau}^{\prime} X^{\tau} \quad|\tau|=m \quad X^{\tau} \prec X^{\alpha} X_{i} \\
F_{\gamma} & :=X^{\gamma}+\sum C_{\gamma \eta} X^{\eta}
\end{aligned}
$$

Note that we use the same names for some of the coefficients that appears in polynomials $F$ and $G$, so that $F_{\alpha n}=X_{n} G_{\alpha}$ and $F_{\gamma}=G_{\gamma}$. Applying the procedure described in Definition 3.4 on the set of polynomials $F$ we obtain an ideal $\mathfrak{h}^{\prime} \subset k\left[C, C^{\prime}\right]$ defining $\mathcal{S} t_{h}\left(\mathfrak{j}_{\geqslant m}, T\right)$.

Thanks to i) we know that $C^{\prime}$ is a set of eliminable variables for $\mathfrak{h}^{\prime}$ and so $\mathcal{S t}_{h}\left(\mathfrak{\mathfrak { j }}_{\geqslant m}, T\right)$ is also defined by $\mathfrak{h}=\mathfrak{h}^{\prime} \cap k[C]$. The statement follows once we show that $\overline{\mathfrak{h}}=\mathfrak{h}$. 
In order to eliminate the variables $C^{\prime}$ we consider every monomial $X^{\alpha} X_{i}=\operatorname{LM}\left(F_{\alpha i}\right)$ and reduce it using the polynomials $G$. In this way we obtain a polynomial $H_{\alpha i} \in(G) k[X, C]$ such that $X^{\alpha} X_{i}+H_{\alpha i}$ is completely reduced w.r.t. j. Then also $X^{\alpha} X_{i} X_{n}+H_{\alpha i} X_{n}+\sum C_{\alpha i \tau}^{\prime} X^{\tau} X_{n}$ (i.e. $F_{\alpha i} X_{n}+H_{\alpha i} X_{n}$ ) is reduced modulo $\dot{j}$ and moreover it belongs to $(F) k\left[X, C, C^{\prime}\right]$ because $X_{n} G \subseteq(F) k\left[X, C, C^{\prime}\right]$. Its $X$-coefficients belong to $\mathfrak{h}^{\prime}$, because the ideal $\mathfrak{h}^{\prime}$ is generated by the $X$-coefficient of the polynomials in $\left.(F) k\left[X, C, C^{\prime}\right]\right)$ that are reduced modulo $\dot{j}_{m-1}$ or modulo $j$, which is the same (Proposition 3.5 ii) and Theorem 3.6). The $X$-coefficients of $F_{\alpha i} X_{n}+H_{\alpha i} X_{n}$ are also the $X$-coefficients of $F_{\alpha i}+H_{\alpha i}$, and are precisely the set of polynomials of the type $C_{\alpha i \tau}^{\prime}-\phi_{\alpha i \tau}(C)$ that allow us to eliminate the variables $C^{\prime}$. So the elimination of $C^{\prime}$ is obtained simply putting $C_{\alpha i \tau}^{\prime}=\phi_{\alpha i \tau}(C)$. In this way $F_{\alpha i}$ becomes $-H_{\alpha i}$ that belongs to $(G) k[X, C]$.

The ideal $\mathfrak{h}$, obtained from $\mathfrak{h}^{\prime}$ eliminating $C^{\prime}$, can also be obtained first eliminating $C^{\prime}$ and after taking $X$-coefficients, because the procedure of eliminating $C^{\prime}$ and that of taking $X$-coefficients commute. So $\mathfrak{h}$ is generated by the $X$-coefficients of polynomials in $\left(X_{n} G_{\alpha},-H_{\alpha i}, G_{\gamma}\right) k[X, C]$ that are reduced modulo $i$.

Hence $\mathfrak{h} \subseteq \overline{\mathfrak{h}}$ because $\left(X_{n} G_{\alpha},-H_{\alpha i}, G_{\gamma}\right) k[X, C] \subset(G) k[X, C]$.

On the other hand, $X_{n}(G) k[X, C]=\left(X_{n} G_{\alpha}, X_{n} G_{\gamma}\right) k[X, C] \subset$ $\left(X_{n} G_{\alpha}, G_{\gamma}\right) k[X, C]$. Moreover two polynomials $Q$ and $X_{n} Q$ have the same $X$-coefficients and either one is reduced modulo $j$ if and only the other is. Hence we obtain the opposite inclusion $\overline{\mathfrak{h}} \subseteq \mathfrak{h}$ and conclude.

iii) We use ii) and prove that in the present hypothesis, $\mathcal{S} t_{h}\left(j_{\geqslant m}\right) \simeq$ $\mathcal{S} t_{h}\left(\dot{j}_{\geqslant m}, T\right)$, where $T$ is defined as in $\left.i i\right)$. Following Definition 3.4, we obtain the ideal $\mathfrak{h}_{m}$ of $\mathcal{S} t_{h}\left(\mathfrak{j}_{\geqslant m}\right)$ using:

$$
\begin{aligned}
F_{\alpha n}^{\prime \prime} & :=X^{\alpha} X_{n}+\sum C_{\alpha \delta} X^{\delta} X_{n}+\sum C_{\alpha \sigma}^{\prime \prime} X^{\sigma}, \quad X_{n} \nmid X^{\sigma} \\
F_{\alpha i} & :=X^{\alpha} X_{i}+\sum C_{\alpha i \tau}^{\prime} X^{\tau} \\
F_{\gamma} & :=X^{\gamma}+\sum C_{\gamma \eta} X^{\eta}=G_{\gamma} .
\end{aligned}
$$

Note that $F_{\alpha i}$ and $F_{\gamma}$ are as in $i i$ ), but all the degree $m$ monomials of the tail of $X_{n} X^{\alpha}$ appear in $F_{\alpha n}^{\prime \prime}$, and not only those divided by $X_{n}$.

For every monomial $X^{\alpha}$ of degree $m-1$ in the basis of $j_{m-1}$, let us consider the $S$-polynomial:

$$
S\left(F_{\alpha n}^{\prime \prime}, F_{\alpha n-1}\right)=\sum C_{\alpha \delta} X^{\delta} X_{n-1} X_{n}+\sum C_{\alpha \sigma}^{\prime \prime} X^{\sigma} X_{n-1}-\sum C_{\alpha i \tau}^{\prime} X^{\tau} X_{n} .
$$

By our hypothesis no monomial appearing in it belongs to $\dot{\mathfrak{I}}_{m}$. In fact $X^{\sigma} X_{n-1} \in j$ if and only if it is a minimal generator of $j$, which is excluded by 
hypothesis because its degree is $m+1$, or it is of the type $X^{\alpha} X_{a}$ with $X^{\alpha}$ minimal generator of $\dot{j}_{m}$ and $X_{a}=\min \left(X^{\sigma} X_{n-1}\right)=X_{n-1}$, while $X^{\sigma} \notin \dot{j}_{m}$. Then $S\left(F_{\alpha n}^{\prime \prime}, F_{\alpha n-1}\right)$ is already reduced with respect to $\dot{j}_{m}$ and so its $X$ coefficients belong to $\mathfrak{h}_{m}$. Especially, as both $X^{\delta} X_{n-1} X_{n}$ and $X^{\tau} X_{n}$ are multiple of $X_{n}$, while $X^{\sigma} X_{n-1}$ is not, the coefficient of $X^{\sigma} X_{n-1}$ is simply $C_{\alpha \sigma}^{\prime \prime}$ so that each $C_{\alpha \sigma}^{\prime \prime}$ belongs to $\mathfrak{h}_{m}$. Hence we can eliminate all the variables $C^{\prime \prime}$, just putting them equal to 0 . In this way $F_{\alpha n}^{\prime \prime}$ becomes $F_{\alpha n}$ as in (4.7) and $\mathcal{S} t_{h}\left(\mathfrak{j}_{\geqslant m}\right) \simeq \mathcal{S} t_{h}\left(\dot{\mathfrak{j}}_{\geqslant m}, T\right)$, where $T$ is as in $\left.i i\right)$, and we conclude because $\mathcal{S} t_{h}\left(\dot{\mathfrak{j}}_{\geqslant m}, T\right) \simeq \mathcal{S} t_{h}\left(\mathfrak{j}_{\geqslant m-1}\right)$.

iv) By $i i)$, we know that ed $\mathcal{S} t_{h}\left(\dot{\mathrm{j}}_{\geqslant m}\right) \geqslant \operatorname{ed} \mathcal{S} t_{h}\left(\mathfrak{j}_{\geqslant m}, T\right)=\mathcal{S} t_{h}\left(\dot{\mathrm{j}}_{\geqslant m-1}\right)$, where the tails defined in $T$ contain only monomials divided by $X_{n}$. Let us now consider a monomial $X^{\alpha}$ among the generators of $j$ of degree smaller than $m$ and a generator $X^{\gamma}$ of degree $m+1$ divided by $X_{n-1}$. Computing the stratum $\mathcal{S} t_{h}\left(\dot{j}_{\geqslant m}\right)$, in the tail of $X^{\alpha} X_{n}^{m-|\alpha|}$ there is the monomial $X^{\beta}=X^{\gamma} / X_{n-1}$ not belonging to $T$. Let us call $D$ the coefficient of $X^{\beta}$, that is

$$
F=X^{\alpha} X_{n}^{m-|\alpha|}+\ldots+D X^{\beta}+\ldots
$$

Thinking about the syzygies of the ideal $j$, it is easy to see that in any $S$-polynomial, $F$ is surely multiplied by a monomial $X^{\delta}$ divided at least by one variable $X_{i}, i<n$. Therefore in every $S$-polynomial the monomial $X^{\beta} X^{\delta}=\left(X^{\beta} X_{i}\right) X^{\delta^{\prime}}$ belongs to $\dot{j}$ because of the Borel-fixed hypothesis, so that it can be reduced. Finally there is no equation involving the variable $D$, so it is free and it cannot be eliminated. Repeating the reasoning for the $M$ minimal generators of degree smaller than $m+1$ and for the $N$ generators divided by $X_{n-1}$ of degree $m+1$, we obtain the thesis.

$v$ ) straightforward applying $i v$ ). vi) straightforward applying $i i i$ ).

With the following examples, we want to underline again the not so crucial role played by term ordering in this construction (Example 4.8) and we want to show (Example 4.9 and Example 4.10) that the estimate of growth of the embedding dimension of the stratum introduced in Theorem $4.7 \mathrm{iv}$ ) is a lower bound.

EXAMPLE 4.8. Let us consider the ideals $\dot{i}=\left(X_{0}, X_{1}^{2}, X_{1} X_{2}\right)$ and $\dot{j}=\dot{i}_{\geqslant 2}=\left(X_{0}^{2}, X_{0} X_{1}, X_{0} X_{2}, X_{0} X_{3}, X_{1}^{2}, X_{1} X_{2}\right)$ in the ring $k\left[X_{0}, X_{1}, X_{2}, X_{3}\right]$ and the strata of the ideal $j$ according to two different term orderings: $\mathcal{S} t_{h}(\dot{\mathfrak{j}}$, Lex $)$ and $\mathcal{S t}_{h}(\dot{\mathrm{j}}$, DegRevLex). In the first case there are at first 24 new variables $C$, whereas in the second case they are 23, so we may guess that the family of the ideals with initial ideal $j$ w.r.t. Lex could be different from the family of the ideals with initial ideal $j$ w.r.t. DegRevLex. 
However applying Theorem 4.7, we can see that $\mathcal{S} t_{h}(\dot{j}$, Lex $) \simeq \mathcal{S} t_{h}(\dot{i}$, Lex $)$ and $\mathcal{S} t_{h}(\mathrm{j}$, DegRevLex $) \simeq \mathcal{S} t_{h}(\mathrm{i}$, DegRevLex $)$. Now the tails of the 3 monomials that generate $\dot{i}$ are the same w.r.t. both term orders and then (see Remark 3.3)

$$
\mathcal{S} t_{h}(\mathrm{j}, \text { Lex }) \simeq \mathcal{S} t_{h}(\mathfrak{i}, \text { Lex })=\mathcal{S} t_{h}(\mathrm{i}, \text { DegRevLex }) \simeq \mathcal{S} t_{h}(\dot{j}, \text { DegRevLex }) .
$$

EXAmPle 4.9. Let us consider the polynomial ring $k\left[X_{0}, X_{1}, X_{2}, X_{3}\right]$, the ideal $\mathrm{j}=\left(X_{0}^{2}, X_{0} X_{1}, X_{0} X_{2}^{4}, X_{1}^{7}, X_{1}^{6} X_{2}^{2}\right)$ and any term ordering given by a matrix with first row $(23,5,2,1)$. By the previous theorem we know that

$$
\begin{gathered}
\mathcal{S} t_{h}(\mathrm{j}) \simeq \mathcal{S} t_{h}\left(\mathrm{j}_{\geqslant 3}\right), \quad \mathcal{S} t_{h}\left(\dot{j}_{\geqslant 4}\right) \simeq \mathcal{S} t_{h}\left(\mathrm{j}_{\geqslant 5}\right) \simeq \mathcal{S} t_{h}\left(\dot{j}_{\geqslant 6}\right), \\
\mathcal{S} t_{h}\left(j_{\geqslant 7}\right) \simeq \mathcal{S} t_{h}\left(\dot{j}_{\geqslant m}\right), \forall m \geqslant 8
\end{gathered}
$$

and

$$
\text { ed } \mathcal{S} t_{h}\left(\mathfrak{j}_{\geqslant 4}\right) \geqslant \operatorname{ed} \mathcal{S} t_{h}(\mathfrak{j})+2 \quad \text { ed } \mathcal{S} t_{h}\left(\mathfrak{j}_{\geqslant 7}\right) \geqslant \operatorname{ed} \mathcal{S} t_{h}\left(\mathfrak{j}_{\geqslant 4}\right)+3
$$

By a direct computation, we find ed $\mathcal{S} t_{h}(\mathfrak{j})=46$, ed $\mathcal{S} t_{h}\left(\dot{\mathfrak{j}}_{\geqslant 4}\right)=50$ and ed $\mathcal{S} t_{h}\left(\dot{j}_{\geqslant}\right)=56$.

EXAMPLE 4.10. There are at most two possible classes of isomorphism for the strata $\mathcal{S t}_{h}\left(\mathfrak{R}_{\geqslant m}\right)$, where $\mathfrak{Q}$ is a lexicographic ideal: $\mathcal{S t}_{h}(\mathfrak{R})$ and $\mathcal{S} t_{h}\left(\Omega_{\geqslant r-1}\right)$, where $r$ is the maximal degree of a minimal generator, in fact the variable $X_{n-1}$ appears (if it does) only in the generator of degree $r$. Called $b$ the number of generators of degree $r$, applying Theorem $4.7 \mathrm{iv}$ ), we have

$$
\operatorname{ed} \mathcal{S} t_{h}\left(\mathfrak{L}_{\geqslant r-1}\right) \geqslant \operatorname{ed} \mathcal{S t}_{h}(\mathfrak{R})+n-b .
$$

If the monomial of maximal degree in the basis does not contain the variable $X_{n-1}$, we have $\mathcal{S} t_{h}\left(\mathfrak{Q}_{\geqslant m}\right) \simeq \mathcal{S} t_{h}(\mathfrak{R}), \forall m$.

We conclude this section with a result similar to the one stated in Theorem 4.7 that concerns only the case of homogeneous Gröbner strata w.r.t. DegRevLex.

Proposition 4.11. Let $\mathrm{j}$ be a Borel-fixed saturated monomial ideal and let $\prec$ be the DegRevLex term ordering. Then

$$
\mathcal{S} t_{h}(\mathrm{j}) \simeq \mathcal{S} t_{h}\left(\dot{\mathrm{j}}_{\geqslant m}\right), \quad \forall m .
$$

Proof. The arguments to achieve the proof are very similar to the arguments used in the proof of Theorem 4.7. First of all let us consider the 
monomials

$$
F_{\alpha}=X^{\alpha}+\sum C_{\alpha \beta} X^{\beta}
$$

corresponding to the monomial basis $B_{\mathfrak{j}}$ of $\mathfrak{j}$ and the ideal $\mathfrak{h}(\mathfrak{j}) \subset k[C]$ of the stratum $\mathcal{S} t_{h}(\mathrm{j})$.

In order to compute $\mathcal{S} t_{h}\left(\dot{\mathfrak{j}}_{\geqslant m}\right)$, we have to consider again polynomials $F_{\alpha}$ as before if $|\alpha| \geqslant m, X^{\alpha} \in B_{\mathrm{j}}$ and new polynomials $G_{\alpha \varepsilon}$ such that $\operatorname{LT}\left(G_{\alpha \varepsilon}\right)=$ $X^{\alpha+\varepsilon}, \forall X^{\alpha} \in B_{\mathrm{j}},|\alpha|<m$, and $\forall X^{\varepsilon}$ of degree $m-|\alpha|$, especially $X^{\alpha} X_{n}^{m-|\alpha|}$. Then by the definition itself of DegRevLex, the tail of $X^{\alpha} X_{n}^{m-|\alpha|}$ contains exactly the monomials in the tail of $X^{\alpha}$ multiplied by $X_{n}^{m-|\alpha|}$. So we can write

$$
G_{\alpha \varepsilon}= \begin{cases}X^{\alpha+\varepsilon}+\sum E_{\alpha \delta}^{\varepsilon} X^{\delta}, & \forall X^{\varepsilon} \neq X_{n}^{m-|\alpha|}, \\ X^{\alpha} X_{n}^{m-|\alpha|}+\sum C_{\alpha \beta} X^{\beta} X_{n}^{m-|\alpha|}=X_{n}^{m-|\alpha|} F_{\alpha}, & \text { if } X^{\varepsilon}=X_{n}^{m-|\alpha|}\end{cases}
$$

hence $\mathfrak{h}\left(\mathfrak{j}_{\geqslant m}\right) \subset k[C, E]$ (note that in the present case variables $D$ do not appear by construction).

By Theorem $4.7 i$ ), we know that all the variables $E$ can be eliminated. By the same reasoning used in the proof of Theorem $4.7 \mathrm{ii}$ ), the ideal $\overline{\mathfrak{h}}=\mathfrak{h}\left(\mathfrak{j}_{\geqslant m}\right) \cap k[C]$ contains the $X$-coefficients of a set of S-polynomials corresponding to a set of the $S$-polynomials of the monomial basis of $j$ : so $\mathcal{S} t_{h}(\mathfrak{j}) \simeq \mathcal{S} t_{h}\left(\dot{\mathfrak{j}}_{\geqslant m}\right)$.

\section{Gröbner strata and regularity.}

In the present and following sections $k[X], \prec$ and $j=\left(X^{\gamma_{1}}, \ldots, X^{\gamma_{t}}\right)$ will be as in the previous, but from now on we will consider only homogeneous ideals (with respect to the usual grading) and $T_{i}$ will be the complete homogeneous tail of $X^{\gamma_{i}}$ so that the only involved strata will be the homogeneous strata $\mathcal{S} t_{h}(\mathfrak{j})$ introduced in Definition $3.2 \mathrm{ii}$ ). Since every tail is fixed by $\prec$, we will simply denote ideals defining Gröbner strata by $\mathfrak{h}(\mathfrak{j})$.

Let $p(z)$ be any admissible Hilbert polynomial for subschemes in $\boldsymbol{P}^{n}$. Our goal is to show that the Hilbert scheme $\mathcal{H i l b}_{p(z)}^{n}$ can be covered by homogeneous strata of the type $\mathcal{S} t_{h}(\mathrm{j})$. In order to prove that, it is convenient to think of $\mathcal{H i l b} b_{p(z)}^{n}$ and $\mathcal{S} t_{h}(\mathrm{j})$ as schemes parameterizing the same kind of objects, namely homogeneous ideals in $k[X]$; as many ideals define the same subscheme $Z \subset \boldsymbol{P}^{n}$, the problem is to select a unique ideal in $k[X]$ for every subscheme $Z$. The most common choice is to associate to $Z$ the only homogeneous saturated ideal $I(Z)$ such that $Z=\operatorname{Proj}(k[X] / I(Z))$; this point of view is that assumed for instance in [19] and in [22], where homogeneous strata of saturated ideals are considered. 
Here we prefer a different approach, that directly calls back to the explicit construction of Hilbert schemes (see for instance [1, 11, 23]).

Definition 5.1. Given an admissible Hilbert polynomial $p(z)$ for subschemes in $\boldsymbol{P}^{n}$, we will denote by $r$ the Gotzmann number of $p(z)$, that is the worst regularity of saturated ideals defining subschemes in $\mathcal{H}$ ilb $b_{p(z)}^{n}$. Moreover we set: $M:=\left(\begin{array}{c}n+r \\ n\end{array}\right), t:=M-p(r), M_{1}:=\left(\begin{array}{c}n+r+1 \\ n\end{array}\right)$ and $t_{1}:=$ $M_{1}-p(r+1)$.

Macaulay's Theorem states that $r$ is the regularity of the lexsegment ideal with Hilbert polynomial $p(z)$ (for the definition and the main properties of regularity and for some consequences, we refer to [10]).

As $Z=\operatorname{Proj}(k[X] / I(Z))=\operatorname{Proj}\left(k[X] / I(Z)_{\geqslant r}\right), Z$ can be uniquely identified by the ideal $I(Z)_{\geqslant r}$, which is generated by $t$ linearly independent degree $r$ homogeneous polynomials $F_{1}, \ldots, F_{t}$ or, more precisely, by the $t$ dimensional $k$-vector space $I(Z)_{r}: \mathcal{H}$ ill ${ }_{p(z)}^{n}$ can be realized as a closed subscheme in the grassmannian of the $t$-dimensional vector spaces in $k[X]_{r}$. A $t$-dimensional vector space in $k[X]_{r}$ gives a point in $\mathcal{H}$ ilb $_{p(z)}^{n}$ if and only if it generates an ideal $i$ having $p(z)$ as Hilbert polynomial.

Notation 5.2. From now on, $\mathfrak{i} \in \mathcal{H}$ ill $b_{p(z)}^{n}$ will mean that $\mathfrak{i}=I(Z)_{\geqslant r}$ for some closed subscheme $Z$ in $\boldsymbol{P}^{n}$ with Hilbert polynomial $p(z)$. Equivalently we can say that $i \in \mathcal{H}$ ill $b_{p(z)}^{n}$ if and only if $i$ is an homogeneous ideal in $k[X]$ with Hilbert polynomial $p(z)$ (for the meaning of "Hilbert polynomial of $i$ " see Notation 6) which is generated in degree $r$, where $r$ is the Gotzmann number of $p(z)$.

REMARK 5.3. If $\mathfrak{i} \in \mathcal{H}$ illo $b_{p(z)}^{n}$, then $\mathfrak{i}$ is $r$-regular and it has a free resolution of the type:

$$
0 \rightarrow k[X](-r-\lambda)^{n_{\lambda}} \rightarrow \ldots \rightarrow k[X](-r-1)^{n_{1}} \rightarrow k[X](-r)^{n_{0}} \rightarrow \mathfrak{i} \rightarrow 0
$$

([6, Theorem 1.2]). Then we can find a set of generators for the first syzygies $\operatorname{Syz}(\mathfrak{i})$ in degree $r+1$.

If we take into consideration the homogeneous Gröbner strata $\mathcal{S} t_{h}(\mathfrak{j})$ and select the monomial ideal $j$ in $\mathcal{H}$ ilb $b_{p(z)}^{n}$, we obtain the intended direct relation between Gröbner strata and Hilbert schemes.

Lemma 5.4. If $\mathrm{j} \in \mathcal{H}$ ill $b_{p(z)}^{n}$, then (at least set-theoretically) $\mathcal{S t}_{h}(\mathrm{j}) \subseteq$ $\mathcal{H}$ ilb $_{p(z)}^{n}$. 
Proof. Let $\mathfrak{i}$ be any ideal in $\mathcal{S} t_{h}(\mathfrak{j})$. By hypothesis $\operatorname{LT}(\mathfrak{i})=\mathfrak{j}$ and then $\mathfrak{i}$ and $j$ share the same Hilbert function. Therefore $i$ is generated in degree $r$ and has Hilbert polynomial $p(z)$ and then $\mathfrak{i} \in \mathcal{H}$ ill $b_{p(z)}^{n}$.

Now we will see that the set-theoretic inclusions are in fact algebraic maps and that for some ideals they are open injections. The crucial point is that the stratum structure (and so its injection in the Hilbert scheme) depends on the ideal $j$ and not on the the corresponding subscheme $Z=\operatorname{Proj}(k[X] / \mathfrak{j})$. This is not so surprising because the choice of the ideal fixes all the allowed deformations, but we want to stress this issue because in [19] the authors underestimated this fact and they made a wrong choice (proof of Corollary 4.4). In fact the stratum of the saturated lexicographic ideal $\mathfrak{Q}$ with Hilbert polynomial $p(z)$ is not in general isomorphic to an open subset of $\mathcal{H}$ ill $b_{p(z)}^{n}$ (see [22] and Example 4.10), whereas, as we will see, the stratum of its truncation $\mathfrak{L}^{\prime}=\mathfrak{L}_{\geqslant}$is an open subset of the Reeves-Stillman component of $\mathcal{H}$ ilb $_{p(z)}^{n}$.

Let $j$ be a monomial ideal in $\mathcal{H}$ ill $b_{p(z)}^{n}$. As seen in $\S 3$ every ideal $i$ such that $\mathrm{LT}(\mathfrak{i})=\mathfrak{j}$ has a (unique) reduced Gröbner basis $\left\{f_{1}, \ldots, f_{t}\right\}$ where $f_{i}$ is as in Definition $3.2 \mathrm{ii}$ ). Not every ideal generated by $t$ polynomials of such a type has $j$ as initial ideal. In order to obtain equations for $\mathcal{S} t_{h}(\mathrm{j})$ we consider the coefficients $c_{i \alpha}$ appearing in the $f_{i}$ as new variables; more precisely let $C=\left\{C_{i \alpha}, i=1, \ldots, t, X^{\alpha} \in k[X]_{r} \backslash \dot{i}_{r}\right.$ and $\left.X^{\alpha} \prec X^{\gamma_{i}}\right\}$ be new variables and consider $t$ polynomials in $k[X, C]$ of the following type:

$$
F_{i}=X^{\gamma_{i}}+\sum_{X^{\alpha} \in T_{i}} C_{i \alpha} X^{\alpha}
$$

where $T_{i}=T_{\gamma_{i}} \cap k[X]_{r}$. (Definition 3.1). We obtain the ideal $\mathfrak{h}(\mathfrak{j})$ of $\mathcal{S}_{h}(\mathfrak{j})$ collecting the $X$-coefficients of some complete reduction with respect to $F_{1}, \ldots, F_{t}$ of all the $S$-polynomials $S\left(F_{i}, F_{j}\right)$, corresponding to a set of generators for Syz(j) (see Theorem 3.6 and Proposition 3.5 v)).

Proposition 5.5. In the above notation, let $\mathrm{j}$ be a monomial ideal in $\mathcal{H}$ ill $b_{p(z)}^{n}$ and let $A$ be the $t(n+1) \times M_{1}$ matrix whose entries are the $X$ coefficients of $X_{j} F_{i}$, for all $j=0, \ldots, n$ and $i=1, \ldots, t$.

Then the ideal $\mathfrak{h}(\mathfrak{j})$ of the homogeneous stratum $\mathcal{S} t_{h}(\mathfrak{j})$ is generated by the $\left(t_{1}+1\right) \times\left(t_{1}+1\right)$ minors of $A$.

Proof. By abuse of notation we write in the same way a polynomial and the rows of its $X$-coefficients. As in Definition 3.4 we consider a 
term order on ${ }^{~} \mathbb{T}_{X, C}$ which is an elimination order of the variables $X$ and coincides with the fixed term ordering $\prec$ on ${ }^{\top} T_{X}$. It is quite evident by elementary arguments of linear algebra, that the ideal $a \subseteq k[C]$, generated by all $\left(t_{1}+1\right) \times\left(t_{1}+1\right)$ minors, does not change if we perform some row reduction on $A$. Let $\mathcal{P}$ be a set of $t_{1}$ rows whose leading terms are a basis of $\dot{j}_{r+1}$. If $X_{h} F_{i} \notin \mathcal{P}$, then it has the same leading term than one in $\mathcal{P}$, say $X_{k} F_{j}$; we can substitute $X_{h} F_{i}$ with $X_{h} F_{i}-X_{k} F_{j}$. In this way the rows not in $\mathcal{P}$ become precisely all the $S$-polynomials $S\left(F_{i}, F_{j}\right)$ that have $X$-degree $r+1$.

At the end of this sequence of row reductions, we can write the matrix as follows:

$$
\left(\begin{array}{c|c}
D & E \\
\hline S & L
\end{array}\right)
$$

where $D$ is a $t_{1} \times t_{1}$ upper-triangular matrix with 1's along the main diagonal, whose rows correspond to $\mathcal{P}$ and whose columns correspond to monomials in $j_{r+1}$.

Using rows in $\mathcal{P}$, we now perform a sequence of rows reductions on the following ones, in order to annihilate all the coefficients of monomials in $\dot{\mathrm{j}}_{r+1}$, that is the entries of the submatrix $S$ : if $a(C)$ is the first non-zero entry in a row not in $\mathcal{P}$ and its column corresponds to the monomial $X^{\gamma} \in \dot{\mathrm{j}}_{r+1}$, we add to this row $-a(C) X_{k} F_{j}$, where $X_{k} F_{j} \in \mathcal{P}$ and $\operatorname{LT}\left(X_{k} F_{j}\right)=X^{\gamma}$. This is nothing else than a step of reduction with respect to $\left\{F_{1}, \ldots, F_{t}\right\}$. At the end of this second turn of rows reductions, we can write the matrix as follows:

$$
\left(\begin{array}{c|c}
D & E \\
\hline 0 & R
\end{array}\right)
$$

where the rows in $(D \mid E)$ are unchanged whereas the rows in $(0 \mid R)$ are the $X$-coefficients of complete reductions of $S$-polynomials in $X$-degree $r+1$. Then $a$ is generated by the entries of $R$ and so $\mathfrak{a} \subset \mathfrak{h}(\mathfrak{j})$.

We can see that this inclusion is in fact an equality taking in mind Remark 5.3 and Proposition $3.5 v$ ): the first one says that $\operatorname{Syz}(\mathfrak{j})$ is generated in degree $r+1$ and the second one that in this case $\mathfrak{h}(\mathfrak{j})$ is generated by the $X$-coefficients of complete reductions of the $S$-polynomials $S\left(F_{i}, F_{j}\right)$ of $X$-degree $r+1$.

The following corollary just express in an explicit way two properties contained in the proof of Proposition 5.5. 
COROLlary 5.6. In the above notation:

- the ideal $\mathfrak{h}(\mathfrak{j})$ is generated by the entries of the submatrix $R$ in (5.4);

- the vector space $L(\mathfrak{j})$ is generated by the entries of the submatrix $L$ in (5.3).

As already said in the Remark 3.3, this theorem shows one more time that Gröbner strata equations are substantially independent of the term ordering, that sets only which monomials can appear in the tails $T_{i}$.

\section{Gröbner strata that are open subsets of an Hilbert scheme.}

In the present section we will prove that every homogeneous Gröbner stratum $\mathcal{S t}_{h}(\mathfrak{j})$, where $j \in \mathcal{H}$ ilb $_{p(z)}^{n}$, can be naturally identified with a locally closed subscheme of $\mathcal{H} \mathrm{ilb}_{p(z)}^{n}$ and that it is an open subset of $\mathcal{H}$ illo $b_{p(z)}^{n}$ if $j$ is generated by the first $t$ monomials in $k[X]$ with respect to the fixed term ordering $\prec$. As a consequence we obtain the main results of the paper about the rationality of some components of $\mathcal{H}$ ill $b_{p(z)}^{n}$.

For the meaning of $p(z), r, t, M, t_{1}, M_{1}$ and $\dot{i} \in \mathcal{H}$ ilb $_{p(z)}^{n}$ we refer to Definition 5.1 and Notation 5.2.

First of all we recall how equations defining $\mathcal{H i l b}_{p(z)}^{n}$ are usually obtained (see for instance $[1,11]$ ). Every ideal $i \in \mathcal{H}$ ilb $b_{p(z)}^{n}$ is generated by the $t$-dimensional vector space $\mathfrak{i}_{r}$. On the other hand, thanks to Gotzmann's Persistence Theorem (see for instance [10, Theorem 3.8]), a $t$-dimensional vector space $V \subset k[X]_{r}$ generates an ideal $\dot{i} \in \mathcal{H}$ ilb $b_{p(z)}^{n}$ if and only if $\operatorname{dim}_{k}\left\langle X_{0} V, \ldots, X_{n} V\right\rangle=t_{1}$.

Therefore $\mathcal{H}$ ilb $b_{p(z)}^{n}$ can be thought as the subscheme of the grassmannian $\mathrm{G}(t, M)$ defined by the previous condition. Moreover by the Plücker embedding of the grassmannian in a projective space $\boldsymbol{P}^{q}, \mathcal{H}$ ilb $b_{p(z)}^{n}$ becomes a closed subscheme (not necessarily irreducible and reduced) of $\boldsymbol{P}^{q}$.

Here we are not interested in finding explicit equations for $\mathcal{H i l b}_{p(z)}^{n}$ in $\boldsymbol{P}^{q}$, but only equations defining each open subset $U \cap \mathcal{H}$ ill $b_{p(z)}^{n}$, where $U$ is the open subset of $\mathrm{Gr}(t, M)$ given by a non-vanishing Plücker coordinate.

DEFINITION 6.1. Thinking of $k[X]_{r}$ as the vector space generated by its monomials, we can identify every Plücker coordinate with a suitable monomial ideal $j$ generated by $t$ monomials of degree $r$. We will denote by $U_{\mathrm{i}}$ and $\mathcal{H}_{\mathrm{i}}$ respectively the open subsets of $\mathrm{G}(t, M)$ and of $\mathcal{H}$ ilb $b_{p(z)}^{n}$ where the Plücker coordinate corresponding to $j$ does not vanish. 
In a natural way $U_{\mathrm{j}}$ is isomorphic to the affine space $\mathrm{A}^{t(M-t)}$. In fact, if $\dot{j}=\left(X^{\gamma_{1}}, \ldots, X^{\gamma_{t}}\right)$, every point in $U_{j}$ is uniquely identified by the reduced, ordered set of generators $\left\langle g_{1}, \ldots, g_{t}\right\rangle$ of the type $g_{i}=X^{\gamma_{i}}+\sum c_{i \alpha} X^{\alpha}$, where $c_{i \alpha} \in k$ and $X^{\alpha}$ is any monomial in $k[X]_{r} \backslash j$. Then we consider on $\mathrm{A}^{t(M-t)}$ the coordinates $C_{i \alpha}$. Note that each $C_{i \alpha}$ naturally corresponds to the Plücker coordinate $j^{\prime}=\left(X^{\gamma_{1}}, \ldots, X^{\gamma_{i-1}}, X^{\alpha}, X^{\gamma_{i+1}}, \ldots X^{\gamma_{t}}\right)$ (but of course not all the Plücker coordinates are of this type).

Now we can mimic the construction of Gröbner strata and obtain the defining ideal of $\mathcal{H}_{\mathrm{j}}$ as a subscheme of $\mathrm{A}^{t(M-t)}$. Let us consider the set of variables $\bar{C}=\left\{C_{i \alpha}, i=1, \ldots, t, X^{\alpha} \in k[X]_{r} \backslash j\right\}$ and $t$ polynomials $G_{1}, \ldots, G_{t}$ in $k[X, \bar{C}]$ of the type:

$$
G_{i}=X^{\gamma_{i}}+\sum C_{i \alpha} X^{\alpha}
$$

and let $B$ be the $(n+1) t \times M_{1}$ matrix whose entries are the $X$-coefficients of the polynomials $X_{j} G_{i}$. Then consider the ideal $\mathfrak{b}(\mathfrak{j}) \subset k[\bar{C}]$ generated by the $\left(t_{1}+1\right) \times\left(t_{1}+1\right)$ minors in $B$.

Proposition 6.2. $\quad \mathfrak{b}(\mathfrak{j})$ is the ideal of $\mathcal{H}_{\mathfrak{j}}$ as a closed subscheme of $\mathrm{A}^{t(M-t)}$.

Proof. Every ideal $\mathfrak{i} \in U_{\mathrm{j}}$ can be obtained from $\left(G_{1}, \ldots, G_{t}\right)$ specializing (in a unique way) the variables $C_{i \alpha}$ to $c_{i \alpha} \in k$. Obviously not all the specializations give ideals $i \in \mathcal{H}_{\mathrm{j}}$, that is with Hilbert polynomial $p(z)$ (more precisely, such that $k\left[X_{0}, \ldots, X_{n}\right] / \mathfrak{i}$ has Hilbert polynomial $\left.p(z)\right)$, because we have to ask both $\operatorname{dim}_{k}\left(\dot{i}_{r}\right)=t$ and $\operatorname{dim}_{k} \dot{i}_{r+1}=t_{1}$ : thanks to Gotzmann's persistence we know that these two necessary conditions are also sufficient.

In the open subset $U_{\mathrm{j}}$ the first condition always holds and the rank of every specialization of $B$ is $\geqslant t_{1}$ by Macaulay estimate of the growth of ideals (see [10, Section 3] or [15, Corollary 5.5.28]). Therefore $\mathcal{H}_{\mathrm{j}}$ is given by the condition $\operatorname{rk}(B) \leqslant t_{1}$.

We can order the set of Plücker coordinates in the following way. We write the $t$ monomials corresponding to each Plücker coordinate in decreasing order with respect to $\prec$; if $\dot{j}_{1}=\left(X^{\alpha_{1}} \succ \ldots \succ X^{\alpha_{t}}\right)$ and $\dot{\mathrm{j}}_{2}=\left(X^{\beta_{1}} \succ \ldots \succ X^{\beta_{t}}\right)$, then $\dot{j}_{1} \succ \dot{\mathrm{j}}_{2}$ if $X^{\alpha_{i}}=X^{\beta_{i}}$ for every $i$ lower than some $s$ and $X^{\alpha_{s}} \succ X^{\beta_{s}}$.

It is now easy to compare, for the same monomial ideal $j \in \mathcal{H}$ ill $b_{p(z)}^{n}$, the Gröbner stratum $\mathcal{S} t_{h}(\mathrm{j})$ and the open subset $\mathcal{H}_{\mathrm{j}}$. We underline that for our purpose it will be sufficient to consider the open subsets $\mathcal{H}_{\mathrm{j}}$ corresponding to monomial ideals $j \in \mathcal{H}$ ill $b_{p(z)}^{n}$, because (scheme-theoretically) they cover $\mathcal{H}$ ilb $b_{p(z)}^{n}$. In fact, if $\mathfrak{i} \in \mathcal{H}$ illb $n_{p(z)}^{n}$, then also $\operatorname{LT}(\mathfrak{i}) \in \mathcal{H} \mathrm{ilb}_{p(z)}^{n}$ and so $\mathfrak{i} \in \mathcal{H}_{L T(\mathfrak{i})}$. 
THEOREM 6.3. Let $p(z)$ be any admissible Hilbert polynomial in $\boldsymbol{P}^{n}$ with Gotzmann number $r$. Let us fix any term ordering $\prec$ on $\mathbb{T}_{X}$.

i) If $\mathrm{j}$ is a monomial ideal in $\mathcal{H i l b}_{p(z)}^{n}$, then $\mathcal{S t}_{h}(\mathfrak{j})$ is naturally isomorphic to the locally closed subscheme of $\mathcal{H i l b}_{p(z)}^{n}$ given by the conditions that the Plücker coordinate corresponding to $j$ does not vanish and the preceding ones vanish.

ii) For every isolated, irreducible component $H$ of $\mathcal{H i l b}_{p(z)}^{n}$, there is a monomial ideal $\dot{\mathrm{j}} \in \mathcal{H}_{\mathrm{ilb}}^{n}{ }_{p(z)}$ such that an irreducible component of Supp $\mathcal{S} t_{h}(\mathfrak{j})$ is an open subset of $\operatorname{Supp~H.~Then~Supp~H~has~an~open~subset~}$ which is a homogeneous affine variety with respect to a non-standard positive grading.

iii) Every smooth irreducible component $H$ of $\mathcal{H i l b}_{p(z)}^{n}$ is rational. The same holds for every smooth, irreducible component of Supp $\mathcal{H}$ ill $b_{p(z)}^{n}$.

Proof. i) We obtain the two affine varieties $\mathcal{S} t_{h}(\mathfrak{j})$ and $\mathcal{H}_{j}$ in a quite similar way (for $\mathcal{S} t_{h}(\mathfrak{j})$ see Proposition 5.5 and for $\mathcal{H}_{\mathfrak{j}}$ see Proposition 6.2). The only difference comes from the definition of the set of polynomials $F_{1}, \ldots, F_{t}$ given in (5.2), leading to equations for $\mathcal{S} t_{h}(\mathfrak{j})$, and the set of polynomials $G_{1}, \ldots, G_{t}$ given in (6.1), leading to equations for $\mathcal{H}_{\mathrm{i}}$ : in $G_{i}$ the sum is over all the degree $r$ monomials $X^{\alpha} \notin j$ whereas in $F_{i}$ we also assume that $X^{\alpha} \prec \mathrm{LT}\left(F_{i}\right)$. Therefore we can think of $\mathcal{S} t_{h}(\mathrm{j})$ as the affine subscheme defined by the ideal $\overline{\mathfrak{h}(\mathfrak{j})}$ in the ring $k[X, \bar{C}]$, where $\bar{C}=\left\{C_{i \alpha} \mid i=0, \ldots, n, \quad X^{\alpha} \in k[X]_{r} \backslash \mathfrak{j}\right\}$ generated by $\mathfrak{h}(\mathfrak{j})$ and by $\left(C_{i \alpha} \mid X^{\alpha} \succ \mathrm{LT}\left(F_{i}\right)\right)$, namely $\overline{\mathfrak{h}(\mathfrak{j})}=\mathfrak{h}(\mathfrak{j}) k[\bar{C}]+(\bar{C} \backslash C)$. Now we can conclude because the Plücker coordinates higher than $j$ vanish if and only if all the $C_{i \alpha}$ such that $X^{\alpha} \succ \operatorname{LT}\left(F_{i}\right)$ vanish.

ii) As $j$ varies among the finite set of the monomial ideals in $\mathcal{H}$ ilb ${ }_{p(z)}^{n}$, the Gröbner strata $\mathcal{S} t_{h}(\mathfrak{j})$ give a set theoretical covering of $\mathcal{H}$ ilb $b_{p(z)}^{n}$ by locally closed subschemes. Then there is a suitable ideal $j$ such that an irreducible component of $\operatorname{Supp}_{\mathcal{S}} t_{h}(\mathfrak{j})$ is an open subset of $H$. We have seen in the previous sections that $\mathcal{S} t_{h}(\mathfrak{j})$ has a structure of homogeneous affine scheme with respect to a non-standard positive grading $\lambda$. Then also its support and the irreducible components of the support are homogeneous (see [2, Section IV.3.3] and [8, Corollary 2.7]).

iii) If $H$ is a smooth, irreducible component of either $\mathcal{H i l b}_{p(z)}^{n}$ or Supp $\mathcal{H i l l}_{p(z)}^{n}$, then it is also reduced. Thanks to the previous item we know that an open subset of $H$ is an affine homogeneous variety with respect to a positive grading. Moreover this open subset is also smooth and so it is isomorphic to an affine space, by Corollary 4.5. 
REMARK 6.4. Let $j$ be a monomial ideal in $\mathcal{H i l b}_{p(z)}^{n}$ and let $\mathfrak{b}(\mathfrak{j}) \subset k[\bar{C}]$ the ideal of $\mathcal{H}_{\mathrm{j}}$. It is possible to define a grading $\lambda^{\prime}$ on $k[\bar{C}]$ such that $\mathfrak{b}(\mathfrak{j})$ becomes homogeneous, by the analogous definition: $\lambda^{\prime}\left(C_{i \alpha}\right)=\gamma_{i}-\alpha$ if $C_{i \alpha}$ appears in $G_{i}$ (6.1). However this grading $\lambda^{\prime}$ is not necessarily positive and so it gives less interesting consequences.

If an irreducible component $H$ of $\mathcal{H}$ ilb $_{p(z)}^{n}$ is also reduced, Theorem 6.3 insures that there is an open subset of $H$ which has the structure of homogeneous variety with respect to a positive grading induced from that of a suitable Gröbner stratum $\mathcal{S} t_{h}(\mathrm{j})$.

On the other hand, in the case of a non-reduced component we only know that the support of a suitable open subset is homogeneous with respect to a positive grading, but this does not imply that the open subset itself is homogeneous.

Now we consider a special case in which we obtain a positive grading on an open subset of an irreducible component of $\mathcal{H}$ illo $b_{p(z)}^{n}$, even if not reduced.

DEFINITION 6.5. Given any term order $\prec$ in $T_{X}$, a $(m, \prec)$-segment is a subset $S$ of $k[X]_{m}$ containing the first $|S|$ monomials of degree $m$ with respect to $\prec$, namely such that:

$$
\forall X^{\beta} \in k[X]_{m}, \forall X^{\gamma} \in S: X^{\beta} \succ X^{\gamma} \Rightarrow X^{\beta} \in S .
$$

An $(m, \prec)$-segment $i d e a l$ is a monomial ideal $j$ which is generated by a $(m, \prec)$-segment.

If $\mathfrak{Z}$ is the saturated lexsegment ideal, then for every $m \geqslant r$ (that is for every $m$ higher than the regularity of $\mathfrak{L})$, the ideal $\mathfrak{L}_{\geqslant m}$ is a ( $m$, Lex)segment ideal. This property does not hold in general if the term ordering is not Lex, so that $j_{\geqslant m}$ could be a $(m, \prec)$-segment ideal and $j_{\geqslant m+1}$ could not be a $(m+1, \prec)$-segment ideal. A trivial case is for instance that of the ideal $\left(X_{0}\right)$ in $k\left[X_{0}, X_{1}, X_{2}\right]$ which is (1, DegRevLex)-segment ideal, whereas $\left(X_{0}\right)_{\geqslant 2}=\left(X_{0}^{2}, X_{0} X_{1}, X_{0} X_{2}\right)$ is not a (2, DegRevLex)-segment ideal, because it contains $X_{0} X_{2}$ and does not contain $X_{1}^{2}$.

The definition of ( $m, \prec$ )-segment ideal is not equivalent, but it is very close to that of extremal ideal given in [24].

COROLlary 6.6. Let $j$ be $(r, \prec)$-segment ideal in the grassmannian $\mathrm{G}(t, M)$.

If $\mathrm{j}$ does not belong to $\mathcal{H i l b}_{p(z)}^{n}$, then the open subset $\mathcal{H}_{\mathrm{i}}$ is empty. 
Proof. Any point $i \in \mathcal{H}_{j}$ should belong to the Gröbner stratum $\mathcal{S} t_{h}(\mathrm{j})$, that is it should share the same Hilbert polynomial of $j$, which is not $p(z)$.

The first of the following examples highlights both that Theorem 6.3 does not hold for a monomial ideal $j$ that belongs to $\mathrm{G}(t, M)$ but not to $\mathcal{H}$ ill $b_{p(z)}^{n}$ and that Corollary 6.6 does not hold for a monomial ideal $j$ in $\mathrm{G}(t, M)$ which is not a segment. Moreover Example 6.8 presents a concrete case of empty $\mathcal{H}_{\mathrm{j}}$ as discussed in the previous corollary.

ExAMPLE 6.7. Let us consider the constant Hilbert polynomial $p(z)=2$ on $\boldsymbol{P}^{2}$. As well known, $\mathcal{H}$ ilb $b_{2}^{2}$ is irreducible of dimension 4 . The monomial ideal $j=\left(X_{0}^{2}, X_{0} X_{1}, X_{1}^{2}, X_{2}^{2}\right)$ is generated by 4 monomials of degree 2, but does not belong to $\mathcal{H}$ ilb $b_{2}^{2}$ because its radical is the irrelevant maximal ideal. However, $\mathcal{H}_{\mathrm{j}}$ is non-empty because it contains for instance all the reduced subschemes given by couples of points $P[1: a: b], Q\left[1: a^{\prime}: b^{\prime}\right] \in \boldsymbol{P}^{2}$ such that $a b^{\prime} \neq a^{\prime} b$. By the way, $\mathcal{S} t_{h}(\mathfrak{j})$ cannot have any common point with $\mathcal{H}_{\text {ilb }}^{2}$.

EXAMPLE 6.8. In the example at the end of $\S 8$ the complete list of Borel ideals in $k\left[X_{0}, X_{1}, X_{2}, X_{3}\right]$ with Hilbert polynomial $p(z)=4 z$ is presented. None of them is the (6, DegRevLex)-segment ideal $j$ containing all the $t=60$ degree 6 monomials except the $p(6)=24$ lowest with respect to the term ordering DegRevLex. As $r=6$ is the Gotzmann number of $4 z$ and $M=84$, then $j$ belongs to $\mathrm{G}(t, M)$ and does not to $\mathcal{H}_{\text {ilb }}^{3}$, (j has constant Hilbert polynomial equal to 24 ). Hence $\mathcal{H}_{\mathfrak{j}}$ is empty.

Corollary 6.9. Let $p(z)$ any admissible Hilbert polynomial in $\boldsymbol{P}^{n}$ with Gotzmann number $r$ and let $H$ be an isolated, irreducible component of $\mathcal{H}$ ill $b_{p(z)}^{n}$.

If $H$ contains a point corresponding to an $(r, \prec)$-segment ideal $\dot{j} \in \mathcal{H}$ ilb ${ }_{p(z)}^{n}$ with respect to some term ordering $\prec$ on $\top^{\top}{ }_{X}$, then $\mathcal{S t}_{h}(\mathrm{j})$ is an open subset of $H$, so that $H$ has an open subset which is an homogeneous affine variety with respect to a non-standard positive grading.

Proof. If $j$ is a $(r, \prec)$-segment ideal, then there are no Plücker coordinates preceding that corresponding to $\mathrm{j}$. Thus $\mathcal{S} t_{h}(\mathrm{j}) \cong \mathcal{H}_{\mathrm{j}}$ (see Theorem 6.3) and so $\mathcal{H}_{\mathrm{j}}$ is an affine homogeneous scheme with respect to a positive grading. 
Corollary 6.10. Let $\mathrm{j} \in \mathcal{H}_{\text {ill }}^{n} \mathrm{p}_{p(z)}$ be $(r, \prec)$-segment ideal and let $H$ be an irreducible component of $\mathcal{H} \mathrm{ilb}_{p(z)}^{n}$ containing $\mathrm{j}$. If either of the following condition holds:

i) $\mathcal{S} t_{h}(\mathrm{j})$ is an affine space,

ii) $\mathrm{j}$ is a smooth point of $\mathcal{S} t_{h}(\mathrm{j})$,

iii) $\mathrm{j}$ is a smooth point of $\mathcal{H}$ ilb $b_{p(z)}^{n}$,

then $H$ is rational.

Proof. Straightforward consequence of the previous result and of Corollary 4.5.

\section{Gröbner stratum of the lexsegment ideal.}

In this paragraph the term ordering $\prec$ will be the lexicographic term ordering Lex.

As a first application to the results obtained in $\S 6$, we take into consideration the lexicographic ideal \&. For every admissible Hilbert function $p(z)$ on $\boldsymbol{P}^{n}, \mathcal{H}$ ilb $b_{p(z)}^{n}$ contains the ideal generated by the first $t$ monomials in degree $r$ with respect to the term ordering Lex. In the paper [20] it is proved that the point of $\mathcal{H i l b}_{p(z)}^{n}$ (usually called lexicographic point) corresponding to the subscheme $\operatorname{Proj} k[X] / \mathcal{R}$ is smooth and Reeves and Stillman get the proof by a computation of the Zariski tangent space dimension. The only component of $\mathcal{H i l b} b_{p(z)}^{n}$ containing the lexicographic point is usually denoted by $H_{R S}$. As a consequence of the quoted result by Reeves and Stillman and of Corollary 6.10, we then obtain:

Corollary 7.1. The Reeves and Stillman component $H_{R S}$ of $\mathcal{H i l b} b_{p(z)}^{n}$ is rational.

However we prefer to present here a new, self-contained proof, in order to explain how our technique can be used as a theoretical, as well as a computational, tool.

First of all, we recall briefly the notation used in [20]. Moving from [16], Reeves and Stillman work with lexicographic saturated ideals of the type:

$$
L\left(a_{0}, \ldots, a_{n-1}\right)=\left(X_{0}^{a_{n-1}+1}, X_{0}^{a_{n-1}} X_{1}^{a_{n-2}+1}, \ldots, X_{0}^{a_{n-1}} \cdots X_{n-2}^{a_{1}} X_{n-1}^{a_{0}}\right) .
$$

Since we are going to prove the same result, we will assume a quite similar notation, but not the same because in this paper we consider ideals generated in degree $r$ instead of saturated ideals. 
Notation 7.2. We refer with $\mathfrak{L}\left(a_{0}, \ldots, a_{n-1}\right)$ to the lexsegment ideal generated by all monomials of degree $r=\sum a_{j}$ that precede (greater than or equal to) the monomial $X_{0}^{a_{n-1}} \cdots X_{n-1}^{a_{0}}$ in the Lex term ordering:

$$
\mathfrak{L}\left(a_{0}, \ldots, a_{n-1}\right)=\left(X_{0}^{r}, X_{0}^{r-1} X_{1}, \ldots, X_{0}^{a_{n-1}} \cdots X_{n-1}^{a_{0}}\right) .
$$

Note that $r$ is precisely the Gotzmann number of the Hilbert polynomial of $\mathfrak{Q}$ (more precisely of $k[X] / \mathfrak{R}$ ).

THEOREM 7.3. The homogeneous Gröbner stratum $\mathcal{S t}_{h}\left(\mathbb{2}\left(a_{0}, \ldots, a_{n-1}\right)\right)$ of the lexicographic ideal $\mathfrak{L}\left(a_{0}, \ldots, a_{n-1}\right) \in \mathcal{H} \mathrm{il}_{p(z)}^{n}$ is isomorphic to an affine space. Therefore the component $H_{R S}$ of $\mathcal{H i l b} b_{p(z)}^{n}$ is rational.

PRoof. Thanks to Corollary 6.10 we obtain the complete statement proving that the homogeneous Gröbner stratum $\mathcal{S} t_{h}\left(\mathbb{2}\left(a_{0}, \ldots, a_{n-1}\right)\right)$ is an affine space, that is showing that a same number is both a lower-bound for its dimension and an upper-bound for its embedding dimension; the first part corresponds to Theorem 4.1 of [20] (here in terms of initial ideals) and the second one corresponds to Theorem 3.3 of [20].

We proceed by induction on the number $n$ of variables and on the Gotzmann number $r$.

In order to obtain an upper-bound for the embedding dimension we look for a maximal set of eliminable variables $C^{\prime} \subset C$, using Criterion 4.6. If $X^{\alpha_{1}} \succ \ldots \succ X^{\alpha_{m}}$ is the monomial basis of the saturation $\mathfrak{l}$ of $\mathfrak{L}\left(a_{0}, \ldots, a_{n-1}\right)$, then we can assume that the polynomials $F_{1}, \ldots, F_{t} \in k[X, C]$ (that we use in order to construct $\mathcal{S} t_{h}\left(\mathcal{Q}\left(a_{0}, \ldots, a_{n-1}\right)\right)$ : see Definition 3.4) are ordered so that $\operatorname{LT}\left(F_{i}\right)=X^{\alpha_{i}} X_{n}^{r-\left|\alpha_{i}\right|}$ for $i=1, \ldots, m$. Thanks to Theorem 4.7 we can start the construction of $C^{\prime}$, putting inside all the variables appearing in $F_{j}$ for every $j>m$.

We divide the proof in 3 steps.

STEP 1. The zero-dimensional case: $\mathcal{S} t_{h}\left(\mathcal{L}\left(a_{0}, 0, \ldots, 0\right)\right) \simeq \mathrm{A}^{n a_{0}}$.

Claim 1I: $\operatorname{dim} \mathcal{S} t_{h}\left(\mathcal{R}\left(a_{0}, 0, \ldots, 0\right)\right) \geqslant n a_{0}$.

Let us denote $\mathfrak{L}\left(a_{0}, 0, \ldots, 0\right)$ by $\mathfrak{R}$. The zero-dimensional scheme $Z$ of $a_{0}$ general points in $\boldsymbol{P}^{n}$ has Gotzmann number $a_{0}$ and Hilbert polynomial $p(z)=a_{0}$. Moreover $\operatorname{LT}\left(I(Z)_{\geqslant a_{0}}\right) \supseteq \mathfrak{Q}$, because for every monomial $X^{\gamma} \succeq X_{1}^{a_{1}}$ we can find some homogeneous polynomial of the type $X^{\gamma}-\sum_{j=1}^{a_{0}} b_{j} X_{n-1}^{a_{0}-j} X_{n}^{j}$ vanishing in the $a_{0}$ points of $Z$ : we can find the $b_{j}$ 's solving a $a_{0} \times a_{0}$ linear system with a Vandermonde associated matrix. As both $\operatorname{LT}\left(I(Z)_{\geqslant a_{0}}\right)$ and $\mathcal{L}$ are generated in degree $a_{0}$, they coincide; so $I(Z)_{\geqslant r} \in \mathcal{S} t_{h}(\mathfrak{R})$ and we conclude since we can choose $Z$ in a family of dimension $n a_{0}$. 
Claim 1II: ed $\mathcal{S t}_{h}\left(\mathfrak{Q}\left(a_{0}, 0, \ldots, 0\right)\right) \leqslant n a_{0}$.

The saturation of $\mathfrak{R}$ is the ideal $\left(X_{0}, X_{1}, \ldots, X_{n-2}, X_{n-1}^{a_{0}}\right)$, which is generated by $n$ monomials; moreover there are only $a_{0}$ monomials of degree $a_{0}$ not contained in 2$)$ : Corollary 4.7 leads to the conclusion.

STEP 2: If $\mathcal{S} t_{h}\left(\mathfrak{Q}\left(0, a_{1}, \ldots, a_{n-1}\right)\right) \simeq \mathrm{A}^{K}$ then $\mathcal{S} t_{h}\left(\mathfrak{Q}\left(a_{0}, a_{1}, \ldots, a_{n-1}\right)\right) \simeq$
$\mathrm{A}^{K+n a_{0}}$.

CLAIM 2I: $\operatorname{dim} \mathcal{S} t_{h}\left(\mathbb{2}\left(a_{0}, a_{1}, \ldots, a_{n-1}\right)\right) \geqslant \operatorname{dim} \mathcal{S} t_{h}\left(\mathbb{R}\left(0, a_{1}, \ldots, a_{n-1}\right)\right)+n a_{0}=$ $K+n a_{0}$.

Let us denote $\mathfrak{L}\left(a_{0}, a_{1}, \ldots, a_{n-1}\right)$ by $\mathfrak{Q}$ and $\mathfrak{R}\left(0, a_{1}, \ldots, a_{n-1}\right)$ by $\mathfrak{L}_{1}$. Let $Y$ be any closed subscheme in $\boldsymbol{P}^{n}$ such that $I(Y)_{\geqslant r} \in \mathcal{S} t_{h}\left(\Omega_{1}\right)$ and consider the set $Z$ of $a_{0}$ points in $\boldsymbol{P}^{n}$. If we choose the $a_{0}$ points in $Z$ general enough, then $I(Z \cup Y)=I(Z) \cdot I(Y)$. Then we conclude thanks to the previous step, as $\operatorname{LT}(I(Z))=\mathfrak{L}\left(a_{0}, 0, \ldots, 0\right)$ and $\mathfrak{R}=\mathfrak{R}_{1} \cdot \mathfrak{L}\left(a_{0}, 0, \ldots, 0\right)$.

CLAIM 2II: ed $\mathcal{S} t_{h}\left(\mathfrak{L}\left(a_{0}, a_{1}, \ldots, a_{n-1}\right)\right) \leqslant \mathrm{ed} \mathcal{S} t_{h}\left(\mathfrak{L}\left(0, a_{1}, \ldots, a_{n-1}\right)\right)+n a_{0}=$ $K+n a_{0}$.

First of all, let us consider all the polynomials $F_{i}$ such that $X_{n}^{r-a_{0}} \mid \operatorname{LT}\left(F_{i}\right)$ and the set of variables $C_{i \beta}$ appearing in them such that $X^{\beta}=X^{\beta_{1}} X_{n}^{r-a_{1}}$ for some monomial $X^{\beta_{1}} \notin \mathfrak{Q}_{1}$ : a multiple of $X^{\beta}$ belongs to $\mathfrak{Q}$ if and only the corresponding multiple of $X^{\beta_{1}}$ belongs to $\mathfrak{\Omega}_{1}$. Then $F_{i}=X_{n}^{r-a_{0}} F_{1 i}+\ldots$, where the $F_{i 1}$ 's are the polynomials that appear in the definition of $\mathcal{S} t_{h}\left(\Omega_{1}\right)$. Using the $S$-polynomials involving couples of such polynomials we see that $L\left(\Omega_{1}\right) \subseteq L(\mathbb{R})$; thus all the variables $C_{i \beta}$ of this type are eliminable, except at most $K=$ ed $\mathfrak{Q}_{1}$ of them.

Moreover, for every $i \leqslant n$ there are $a_{0}$ variables $C_{i \beta}$ such that $X^{\beta} \notin \mathbb{R}$, $X^{\beta} \in \mathfrak{R}_{1}$ : the are $X_{0}^{a_{n-1}} \cdots X_{n-2}^{a_{1}} X_{n-1}^{a_{0}-j} X_{n}^{j}, j=1, \ldots, a_{0}$.

If we specialize to 0 all the variables of the two above considered types, the embedding dimension drops at most by ed $\mathcal{S} t_{h}\left(\Omega_{1}\right)+n a_{0}=K+n a_{0}$.

Now it will be sufficient to verify that all the remaining variables $C_{i \beta}$ are eliminable, using Criterion 4.6.

Assume that $X^{\beta} \prec X_{0}^{a_{n-1}} \cdots X_{n-2}^{a_{1}}$ and $X_{n}^{r-a_{0}} \nmid X^{\beta}$.

- If $i>n$, all the variables are eliminable using those appearing in $F_{1}, \ldots, F_{n}$, thanks to Corollary 4.7 .

- If $i<n$, using $S\left(F_{i}, F_{j}\right)$, where $\operatorname{LT}\left(F_{j}\right)=X^{\alpha_{i}} X_{n-1}^{r-\left|\alpha_{i}\right|}$, we see that $C_{i \beta} \in L(\Omega)$. 
- If $i=n$, using $S\left(F_{n}, F_{n-1}\right)=X_{n-2} X_{n}^{a_{0}-1} F_{n}-X_{n-1}^{a_{0}} F_{n-1}$, we see that $C_{n \beta} \in L(\mathcal{L})$ (note that by the previous idem $C_{n-1, \beta^{\prime}} \in L(\mathfrak{L})$ ).

STEP 3: If $\mathfrak{L}\left(0, a_{1}, \ldots, a_{n-1}\right) \simeq \mathrm{A}^{K_{1}}$ then $\mathfrak{L}\left(0, a_{1}, \ldots, a_{d}\right) \simeq \mathrm{A}^{K_{2}}$ where $d$ is the maximal index $<n$ such that $a_{d} \neq 0$ and $K_{2}=K_{1}+(n-d)(d+1)+$ $\left(\begin{array}{c}a_{n-1}+n \\ n\end{array}\right)-1$ (or $K_{2}=K_{1}+\left(\begin{array}{c}a_{n-1}+n \\ n\end{array}\right)-1$ if $d$ does not exist).

Here we compare the ideal $\mathfrak{R}=\mathfrak{L}\left(0, a_{1}, \ldots, a_{n-1}\right)$ in $k[X]$ and the ideal $\mathfrak{\Omega}_{1}=\mathfrak{\Omega}\left(0, a_{1}, \ldots, a_{d}\right)$ in $k\left[X_{0}, \ldots, X_{d}\right]$. Observe that both $\mathfrak{l}:=\operatorname{sat}(\mathfrak{\Omega})$ and $\mathfrak{l}_{1}:=\operatorname{sat}\left(\mathfrak{Q}_{1}\right)$ fulfill the hypothesis of Theorem $4.7 \mathrm{ii}$ ) (see also Example 4.10); then it holds $\mathcal{S} t_{h}(\mathfrak{Q}) \simeq \mathcal{S} t_{h}(\mathfrak{l})$ and $\mathcal{S} t_{h}\left(\mathfrak{Q}_{1}\right) \simeq \mathcal{S} t_{h}\left(\mathfrak{l}_{1}\right)$. The statement for the saturated ideals $\mathfrak{l}$ and $\mathfrak{l}_{1}$ is proved using the same technique as above in [22, Proposition 4.5].

\section{Algorithms and Examples.}

In this final section we exhibit a pseudo-code description of the algorithms to compute the ideal of Gröbner stratum (Algorithm 1) and its embedding dimension (Algorithm 2) and then we apply our technique to the Hilbert scheme $\mathcal{H}$ ilb ${ }_{4 z}^{3}$.

\section{Algorithms.}

The following two algorithms are mainly based on Proposition 5.5 and Corollary 5.6.

Algorithm 1 Computing the ideal $\mathfrak{h}(\mathfrak{j})$ of $\mathcal{S} t_{h}(\mathfrak{j})$

1: Procedure GröbnerStratum $(\mathfrak{j}, \prec)$

2: $\quad$ Compute $B_{\mathrm{j}}$ minimal monomial basis of $\mathrm{j}$;

3: $\mathcal{G} \leftarrow \emptyset$;

4: $\quad$ for all $X^{\alpha} \in B_{\mathrm{j}}$ do

5: $\quad$ Compute $F_{\alpha}=X^{\alpha}+\sum C_{\alpha \beta} X^{\beta}, \quad X^{\beta} \notin \dot{j}, X^{\alpha} \succ X^{\beta},|\alpha|=|\beta|$;

6: $\mathcal{G} \leftarrow \mathcal{G} \cup\left\{F_{\alpha}\right\} ;$

7: $\quad$ end for

8: $\quad$ Compute $\operatorname{Syz}(\mathfrak{j})$ basis of the syzygies of $B_{\mathfrak{i}}$;

9: $\quad \mathcal{C} \leftarrow \emptyset$;

10: $\quad$ for all $\left(\ldots, X^{\delta_{i}}, \ldots, X^{\delta_{j}}, \ldots\right) \in \operatorname{Syz}(\mathfrak{j})$ do

11: $\quad S \leftarrow X^{\delta_{i}} F_{\alpha_{i}}-X^{\delta_{j}} F_{\alpha_{j}}$;

12: $\quad$ Compute the reduction $S_{\text {red }}$ of $S$ w.r.t. the set of polynomials $\mathcal{G}$;

13: $\quad$ Collect the set $\mathcal{R}$ of the $X$-coefficients of $S_{\text {red }}$; 


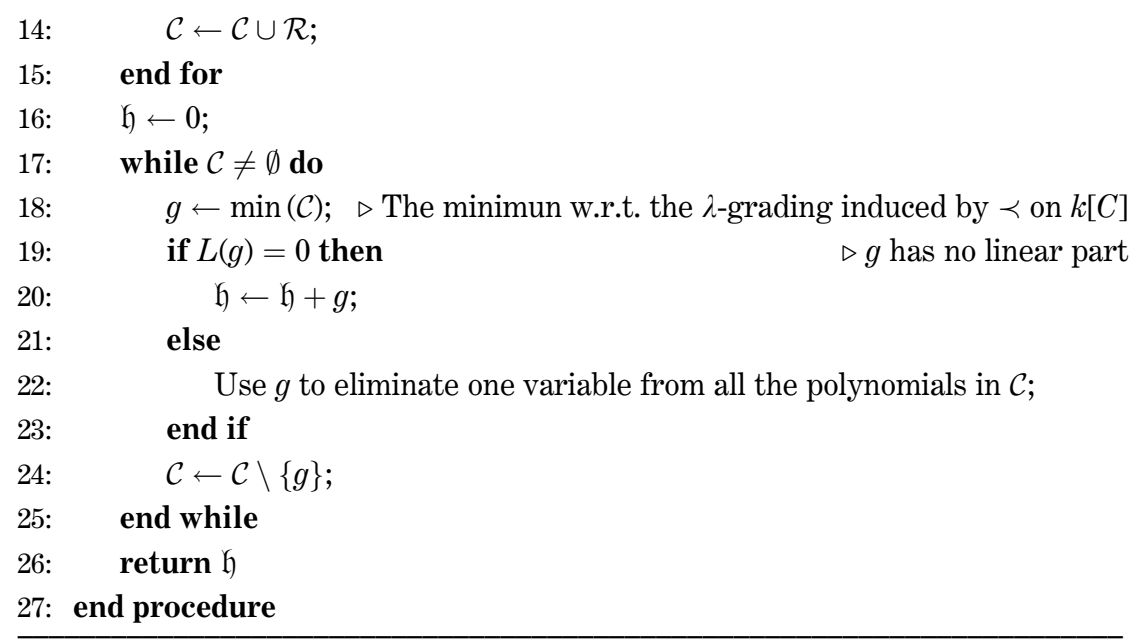

ALGORITHM 2 Computing the embedding dimension of $\mathcal{S} t_{h}(\mathrm{j})$

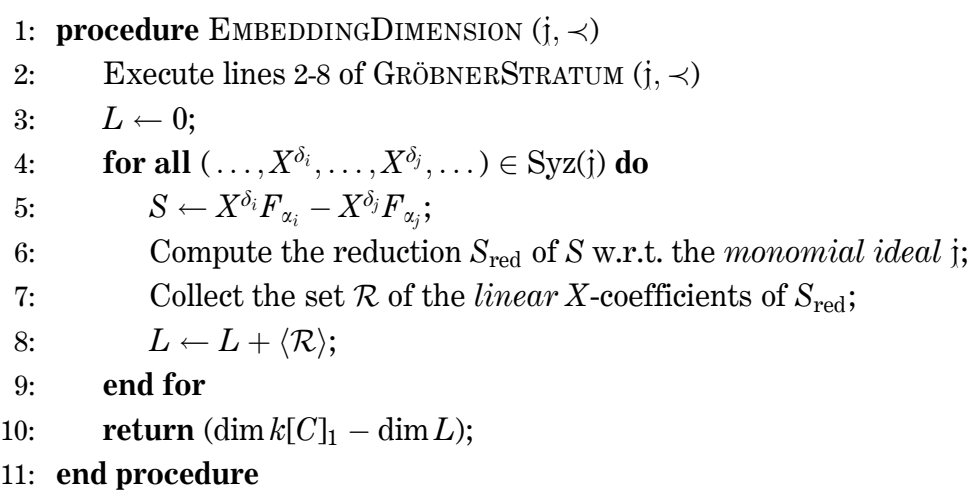

EXAMPLE: $\mathcal{H}$ ilb ${ }_{4 z}^{3}$

In [9], Gotzmann consider the complete list of the Borel-fixed, saturated, monomial ideals of $k\left[X_{0}, X_{1}, X_{2}, X_{3}\right]$ corresponding to points of $\mathcal{H}$ illb ${ }_{4 z}^{3}$. They are:

$$
\begin{aligned}
& \mathfrak{b}_{3}=\left(X_{0}^{2}, X_{0} X_{1}, X_{1}^{3}\right), \\
& \mathfrak{b}_{4}=\left(X_{0}^{2}, X_{0} X_{1}, X_{0} X_{2}^{2}, X_{1}^{4}\right), \\
& \mathfrak{b}_{5}=\left(X_{0}^{2}, X_{0} X_{1}, X_{0} X_{2}, X_{1}^{5}, X_{1}^{4} X_{2}\right), \\
& \mathfrak{b}_{6}=\left(X_{0}, X_{1}^{5}, X_{1}^{4} X_{2}^{2}\right) .
\end{aligned}
$$


The index $s$ in $\mathfrak{b}_{s}$ is the regularity of the ideal. Moving from this point, Gotzmann proves that there are two irreducible components: the first containing $\mathfrak{b}_{3}$ with dimension 16 and the second (the Reeves-Stillman one) containing $\mathfrak{b}_{6}$ with dimension 23 . Here we obtain a computational confirmation of this result. Furthermore we also prove that the two components are reduced, rational and that they have a transversal intersection.

Since the Gotzmann number of the Hilbert polynomial $p(z)=4 z$ is 6 , to deduce informations about $\mathcal{H} \mathrm{ilb}_{4 z}^{3}$ using the results obtained in $\S 6$, we have to consider the truncated ideals $\dot{j}_{s}=\left(\mathfrak{b}_{s}\right)_{\geqslant 6}$.

In the case chark $=0$, a Borel ideal $i$ is characterized by the combinatorial property

$$
X_{i} X^{\alpha} \in \mathfrak{i} \Longrightarrow X_{i-1} X^{\alpha} \in \mathfrak{i} .
$$

As shown in [25], the set of monomials of a fixed degree of a Borel ideal $i$ is a filter for the transitive closure of the partial ordering $\leq_{B}$ induced by the relation (8.1) $\left(X^{\alpha} X_{i-1}>_{B} X^{\alpha} X_{i}\right)$. For each $s$, we can look for a term ordering $\prec_{s}$, obtained refining the partial order $\leq_{B}$, such that the ideal $\dot{j}_{s}$ becomes a $\left(6, \prec_{s}\right)$-segment.

It is possible to achieve this result considering a term ordering given by a matrix of the type:

$$
\left(\begin{array}{llll}
1 & 1 & 1 & 1 \\
a & b & c & d \\
0 & 1 & 0 & 0 \\
0 & 0 & 1 & 0
\end{array}\right)
$$

More precisely:

- $\dot{j}_{3}$ is a segment w.r.t. $\prec_{3}$ given by $(a, b, c, d)=(3,2,1,1)$;

- $\dot{\mathrm{j}}_{4}$ is a segment w.r.t. $\prec_{4}$ given by $(a, b, c, d)=(15,5,2,1)$;

- $\dot{j}_{5}$ is a segment w.r.t. $\prec_{5}$ given by $(a, b, c, d)=(9,3,2,1)$;

- $\dot{\mathrm{j}}_{6}$ is a segment w.r.t. $\prec_{6}$ given by $(a, b, c, d)=(1,0,0,0)$;

Let us now examine these ideals one at the time.

$\dot{j}_{6}$. It is the lexsegment ideal $\mathbb{R}(2,4,0)$, that is $\prec_{6}$ is DegLex (or Lex, which is the same for homogeneous ideals). In the previous section we proved that the Gröbner stratum $\mathcal{S t}_{h}(\mathfrak{R}(2,4,0)$, Lex $)$ is an open subset of $\mathcal{H}$ illb ${ }_{4 z}^{3}$ and that it is an affine space; an easy computation gives the dimension 23.

$\dot{j}_{3}$. As $X_{2}$ does not appear in the monomials of the basis of $\mathfrak{b}_{3}$, thanks to Theorem 4.7, we know that $\mathcal{S} t_{h}\left(\mathfrak{j}_{3}, \prec_{3}\right) \simeq \mathcal{S} t_{h}\left(\mathfrak{b}_{3}, \prec_{3}\right)$. Using a computer 
procedure based on Algorithm 1 we obtain explicit equations for $\mathcal{S t}_{h}\left(\mathfrak{b}_{3}, \prec_{3}\right)$, finding that it is an affine space of dimension 16 , which is isomorphic to an open subset of the component named $H_{V A}$ by Gotzmann (after Vainsencher-Avritzer [26]).

$\dot{\mathfrak{j}}_{4}$. Always by Theorem 4.7 , we can compute $\mathcal{S} t_{h}\left(\mathfrak{b}_{4}, \prec_{4}\right)$ instead of $\mathcal{S} t_{h}\left(\dot{j}_{4}, \prec_{4}\right)$. Using the same computer procedure as below, we compute that initially there are 44 variables $C$, but 20 of them can be eliminated and the minimal embedding realizes $S t_{h}\left(\left(\mathfrak{b}_{4}\right), \prec_{4}\right)$ as an affine subscheme $S$ of $\mathbb{A}^{24}$ given by an ideal $\mathfrak{h}$. Moreover $\mathfrak{h}$ is the product of a principal ideal $(K)$ (more precisely $K$ is the coefficient of the monomial $X_{1}^{3}$ in the polynomial $F_{i}$ such that $\left.\mathrm{LT}\left(F_{i}\right)=X_{0} X_{2}^{2}\right)$ and an ideal $\mathfrak{h}_{1}$. The ideal $(K)$ defines an hyperplane in $\mathrm{A}^{24}$, which is an open subscheme of the $H_{R S}$ component (its dimension is 23). The other ideal $\mathfrak{h}_{1}$ defines an open subscheme of $H_{A V}$. Looking at $\mathfrak{h}_{1}$ it is possible to see that there are some more eliminable variables and that the minimal embedding gives an isomorphism with $\mathrm{A}^{16}$. Looking at the ideals $(K)$ and $\mathfrak{h}_{1}$, it is easy to check that they have a transversal intersection that is the hyperplane defined by $K$ does not contain the Zariski tangent space to $H_{A V}$ at each point in $H_{R S} \cap H_{A V}$.

$\dot{\mathfrak{I}}_{5}$. Applying Theorem 4.7, we compute the structure of the open subset $\mathcal{H}_{\mathfrak{b}_{5}}$ of $\mathcal{H}$ ilb $b_{4 z}^{3}$ by the computation of $\mathcal{S t}_{h}\left(\left(\mathfrak{b}_{5}\right)_{\geqslant 4}, \prec_{5}\right)$. In this case there are 344 new variables $C: 317$ are eliminable, so that the embedding dimension is 27 , that is the point corresponding to $\mathfrak{b}_{5}$ is singular in the Hilbert scheme $\mathcal{H i l b}_{4 z}^{3}$. Going through the computation, we find a Gröbner basis of the ideal $\mathfrak{h}\left(\left(\mathfrak{b}_{5}\right)_{\geqslant 4}\right)$ defined by 9 polynomials. The stratum is irreducible (and so there are no new components): indeed the open subset obtained excluding the hyperplane defined by the coefficient of the monomial $X_{1}^{4}$ of the polynomial with leading term $X_{0} X_{2} X_{3}^{2}$ is isomorphic to an open subset of an affine space of dimension 23, that is to an open subset of the Reeves-Stillman component. On the other hand cutting the stratum with this same hyperplane we obtain an equi-dimensional subscheme of dimension 22 and the same degree than $\mathcal{S} t_{h}\left(\left(\mathfrak{b}_{5}\right)_{\geqslant 4}, \prec_{5}\right)$, which is scheme theoretically the union of two irreducible components $V_{1}$ and $V_{2}$, with the same Hilbert polynomial. One of them can be naturally identified with the stratum of the saturated ideal $\mathfrak{b}_{5}$ in the sense that they are isomorphic and moreover their points correspond to the same curves in $\boldsymbol{P}^{3}$ (see Theorem $4.7 \mathrm{ii}$ )): this component is obtained cutting $\mathcal{S} t_{h}\left(\left(\mathfrak{b}_{5}\right)_{\geqslant 4}, \prec_{5}\right)$ with the hyperplanes defined by the coefficients of $X_{1}^{4}$ in the polynomials with leading term $X_{0} X_{2} X_{3}^{2}, X_{0} X_{1} X_{3}^{2}$ and $X_{0}^{2} X_{3}^{2}$ respectively (see Theorem $4.7 \mathrm{iv}$ ) and $v$ )). The other one $V_{2}$ can be obtained 
from $V_{1}$ up to a special change of coordinates in $\boldsymbol{P}^{3}$. Finally we can verify that the singular locus of $\mathcal{S} t_{h}\left(\left(\mathfrak{b}_{5}\right)_{\geqslant 4}, \prec_{5}\right)$ is contained in the intersection of $V_{1}$ and $V_{2}$.

\section{REFERENCES}

[1] D. BAYER, The division algorithm and the Hilbert schemes. Harvard University, 1982, Ph.D. Thesis.

[2] N. Bourbaki, Commutative algebra. Chapters 1-7. Elements of Mathematics (Berlin). Springer-Verlag, Berlin, 1989. Translated from the French, Reprint of the 1972 edition.

[3] G. Carrà Ferro, Gröbner bases and Hilbert schemes. I. J. Symbolic Comput., 6 (2-3) (1988), pp. 219-230. Computational aspects of commutative algebra.

[4] D. Cox - J. LitTle - D. O'SheA, Ideals, varieties, and algorithms. Undergraduate Texts in Mathematics. Springer, New York, third edition, 2007. An introduction to computational algebraic geometry and commutative algebra.

[5] D. Eisennud, Commutative algebra, volume 150 of Graduate Texts in Mathematics. Springer-Verlag, New York, 1995. With a view toward algebraic geometry.

[6] D. EISENBUD - S. Goto, Linear free resolutions and minimal multiplicity. J. Algebra, 88 (1) (1984), pp. 89-133.

[7] P. ElliA, D'autres composantes non réduites de Hilb $\mathbf{P}^{3}$. Math. Ann., 277 (3) (1987), pp. 433-446.

[8] G. Ferrarese - M. RogGero, Homogeneous varieties for Hilbert schemes. Int. J. Algebra, 3 (9-12) (2009), pp. 547-557.

[9] G. Gotzmann, The irreducible components of $\mathrm{Hilb}^{4 n}\left(P^{3}\right)$, 2008. Preprint.

[10] M. L. Green, Generic initial ideals. pp. 11-85. C.R.M., Bellaterra, 1996. Lectures given at Summer School on Commutative Algebra.

[11] M. Haiman - B. Sturmfels, Multigraded Hilbert schemes. J. Algebraic Geom., 13 (4) (2004, pp. 725-769.

[12] A. Iarrobino, Reducibility of the families of 0-dimensional schemes on a variety. Invent. Math., 15 (1972), pp. 72-77.

[13] A. A. Iarrobino, Punctual Hilbert schemes. Mem. Amer. Math. Soc., 10 (188) (1977), pp. viii +112.

[14] J. O. KLEPPE, Nonreduced components of the Hilbert scheme of smooth space curves. In Space curves (Rocca di Papa, 1985), volume 1266 of Lecture Notes in Math. (Springer, Berlin, 1987), pp. 181-207.

[15] M. Kreuzer - L. RobBiano, Computational commutative algebra. 2. Springer-Verlag, Berlin, 2005.

[16] F. L. MaCaUlay, Some properties of enumeration of modular forms system. Proc. London Math. Soc., 26 (1927), pp. 531-555.

[17] M. G. Marinari - L. Ramella, Some properties of Borel ideals. J. Pure Appl. Algebra, 139 (1-3) (1999), pp. 183-200. Effective methods in algebraic geometry (Saint-Malo, 1998).

[18] D. Mumford, Further pathologies in algebraic geometry. Amer. J. Math., 84 (1962), pp. 642-648. 
[19] R. Notari - M. L. Spreafico, A stratification of Hilbert schemes by initial ideals and applications. Manuscripta Math., 101 (4) (2000), pp. 429-448.

[20] A. Reeves - M. StIllman, Smoothness of the lexicographic point. J. Algebraic Geom., 6 (2) (1997), pp. 235-246.

[21] L. Robbiano, On Border Basis and Groebner Basis Schemes. Collect. Math., 60 (2009), pp. 11-25.

[22] M. Roggero - L. Terracini, Ideals with an assigned initial ideal, Int. Math. Forum, 5 (53-56) (2010), pp. 2731-2750.

[23] E. SERnesi, Deformations of algebraic schemes, volume 334 of Grundlehren der Mathematischen Wissenschaften [Fundamental Principles of Mathematical Sciences]. Springer-Verlag, Berlin, 2006.

[24] M. Sherman. A local version of Gotzmann's Persistence, 2007. Preprint.

[25] M. Sherman, On an extension of Galligo's theorem concerning the Borelfixed points on the Hilbert scheme. J. Algebra, 318 (1) (2007), pp. 47-67.

[26] I. VAinsencher - D. Avritzer, Compactifying the space of elliptic quartic curves. In Complex projective geometry (Trieste, 1989/Bergen, 1989), volume 179 of London Math. Soc. Lecture Note Ser., pp. 47-58. Cambridge Univ. Press, Cambridge, 1992.

Manoscritto pervenuto in redazione il 13 luglio 2010. 